\title{
CATALYTIC ENANTIOSELECTIVE HYDROGENATION OF VINYL BIS(BORONATES).
}

\author{
Jeremy B. Morgan and James P. Morken* \\ Department of Chemistry, Venable and Kenan Laboratories \\ University of North Carolina, Chapel Hill, NC 27599-3290 \\ Supplementary Material
}

General. ${ }^{1} \mathrm{H}$ NMR spectra were recorded on Bruker DRX (400 MHz or $\left.300 \mathrm{MHz}\right)$. Chemical shifts are reported in ppm from tetramethylsilane with the solvent resonance as the internal standard $\left(\mathrm{CDCl}_{3}: 7.24 \mathrm{ppm}\right)$. Data are reported as follows: chemical shift, integration, multiplicity $(\mathrm{s}=$ singlet, $\mathrm{d}=$ doublet, $\mathrm{t}=$ triplet, $\mathrm{q}=$ quartet, $\mathrm{br}=$ broad, $\mathrm{m}=$ multiplet $)$, coupling constants $(\mathrm{Hz})$ and assignment. ${ }^{13} \mathrm{C}$ NMR were recorded on a Bruker DRX $400(100 \mathrm{MHz})$ spectrometer with complete proton decoupling. Chemical shifts are reported in ppm from tetramethylsilane with the solvent as the internal standard $\left(\mathrm{CDCl}_{3}: 77.0 \mathrm{ppm}\right)$. High resolution mass spectrometry was performed by the University of Minnesota Mass Spectrometry Service Laboratory. Infrared (IR) spectra were obtained using a ASI ReactIR 1000.

Liquid chromatography was performed using forced flow (flash chromatography) on silica gel $\left(\mathrm{SiO}_{2}, 32\right.$ to $\left.63 \mu \mathrm{m}\right)$ purchased from Scientific Adsorbents, Inc. Thin layer chromatography (TLC) was performed on EM science $0.25 \mathrm{~mm}$ silica gel 60 plates. Visualization was achieved UV light or phosphomolybdic acid in ethanol followed by heating.

Analytical gas-liquid chromatography (GLC) was performed on a Hewlett-Packard 6890 Series chromatograph equipped with a CTC Analysis Combi Pal autosampler by Leap Technologies (Carrboro, NC), a split mode capillary injection system, a flame ionization detector and a Supelco $\beta$-dex 120 column with helium as the carrier gas. Analytical supercritical fluid chromatography (SFC) was performed on a Berger Instruments supercritical fluid chromatograph equipped with an Alcott autosampler and a Knauer UV detector.

All reactions were conducted in oven and flame dried glassware under an inert atmosphere of argon or hydrogen. All solvents were purged with Argon for a minimum of 15 minutes before use. Toluene was passed through activated basic alumina. Dichloroethane was sequentially washed with concentrated $\mathrm{H}_{2} \mathrm{SO}_{4}$, water, aqueous $\mathrm{Na}_{2} \mathrm{CO}_{3}$, and water, then dried with $\mathrm{MgSO}_{4}$ and fractionally distilled from $\mathrm{CaH}_{2}$. Tetrahydrofuran was fractionally distilled from sodium and benzophenone. Bis(norbornadiene)rhodium(I) tetrafluoroborate was purchased from Alfa Aesar. cis-1,2-bis(4,4,5,5tetramethyl-1,3,2-dioxaborolan-2-yl) styrene, cis-1,2-bis(4,4,5,5-tetramethyl-1,3,2-dioxaborolan-2-yl) heptene, ethylenebis(triphenylphosphine)platinum (0), bis(pinacolato)diboron, anhydrous dimethylformamide, n-butyl lithium in hexanes, bromochloromethane, and all alkynes were purchased from Aldrich Chemical Company. Walphos ligands, tetrakis(triphenylphosphine)platinum (0), and other chiral ligands were purchased from Strem Chemical Company. BoPhoz is commercially available from Alfa Aesar. All reagents were used as received.

Representative Procedure for Catalytic, Enantioselective Hydrogenation Reaction: An ovendried 2 dram vial equipped with a stir-bar was charged with $1.5 \mathrm{mg}(0.004 \mathrm{mmol})$ of bis(norbornadiene)rhodium(I) tetrafluoroborate, $7.4 \mathrm{mg}(0.008 \mathrm{mmol})$ of $(R)$-Walphos (W001), and 1.0 $\mathrm{mL}$ of toluene under an inert atmosphere of argon in a dry-box. The resultant organge-yellow solution 
was stirred for 10 minutes. After this time, $71 \mathrm{mg}(0.2 \mathrm{mmol})$ of cis-1,2-bis(4,4,5,5-tetramethyl-1,3,2dioxaborolan-2-yl) styrene was added to the solution under argon. The vial was transferred to a Parr hydrogenation bomb. The vessel was charged 3 times to 20 bar with $\mathrm{H}_{2}$ gas. Finally, the vessel was charged to 20 bar and the reaction was stirred for 24 hours. After this time, the reaction mixture was diluted with $1.5 \mathrm{~mL}$ of tetrahydrofuran. $0.8 \mathrm{~mL}$ of $3 \mathrm{M} \mathrm{NaOH}$ and $0.4 \mathrm{~mL}$ of $30 \% \mathrm{H}_{2} \mathrm{O}_{2}$ (dropwise with caution) were added, respectively. The mixture was allowed to stir at ambient temperature for 3 hours. The solution was then quenched with $2 \mathrm{~mL}$ of saturated aqueous $\mathrm{Na}_{2} \mathrm{~S}_{2} \mathrm{O}_{3}$. The two-phase mixture was extracted with ethyl acetate $(2 \times 20 \mathrm{~mL})$ and the combined organic layers were washed with brine $(1 \mathrm{x}$ $10 \mathrm{~mL}$ ). The organic layers were then dried over anhydrous $\mathrm{Na}_{2} \mathrm{SO}_{4}$, filtered, and the solvent removed by rotary evaporation. The crude material was purified by silica gel chromatography $(2: 1$ hexanes : ethyl acetate) to provide $23 \mathrm{mg}(84 \%)$ of pure $(S)$-phenyl-ethane-1, 2-diol.

\section{Ligand Structures:}

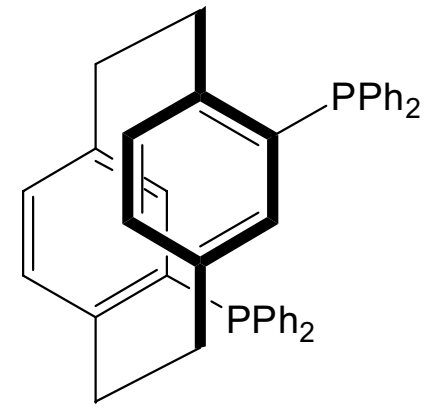

Phanephos

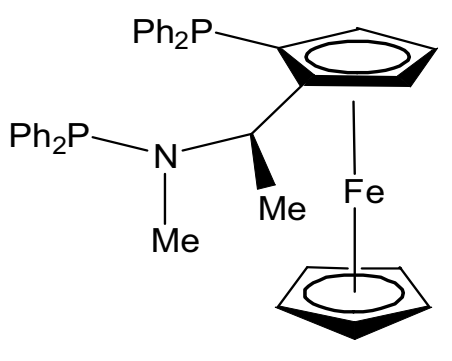

BoPhoz

Representative Procedure for a One-Pot Catalytic Alkyne Diboration/Enantioselective Hydrogenation/Oxidation Reaction: An oven-dried $20 \mathrm{~mL}$ vial equipped with a stir-bar was charged with $7.5 \mathrm{mg}$ (0.01 mmol of ethylenebis(triphenylphosphine)platinum (0), $51 \mathrm{mg}(0.2 \mathrm{mmol})$ of bis(pinacolato)diboron, and $0.5 \mathrm{~mL}$ of toluene under an inert atmosphere of argon in a dry-box. The resulting clear solution was stirred for 1 minute. After this time, $25 \mathrm{mg}(0.24 \mathrm{mmol})$ of phenylacetylene was added to the solution under argon. The vial was sealed and the yellow solution was heated to 100 ${ }^{\circ} \mathrm{C}$ for 48 hours. After this time, an oven-dried 2 dram vial equipped with a stir-bar was charged with $3.7 \mathrm{mg}(0.01 \mathrm{mmol})$ of bis(norbornadiene)rhodium(I) tetrafluoroborate, $13.0 \mathrm{mg}(0.014 \mathrm{mmol})$ of $(R)$ Walphos (W001), and $0.5 \mathrm{~mL}$ of toluene under an inert atmosphere of argon in a dry-box. The resultant organge-yellow solution was stirred for 10 minutes. The crude diboration mixture was transferred under argon to the 2 dram reaction vial using an extra $0.2 \mathrm{~mL}$ of dry toluene for complete transfer. The vial was transferred to a Parr hydrogenation bomb. The vessel was charged 3 times to 20 bar with $\mathrm{H}_{2}$ gas. Finally, the vessel was charged to 20 bar and the reaction was stirred for 24 hours. After this time, the reaction mixture was diluted with $1.5 \mathrm{~mL}$ of tetrahydrofuran. $0.8 \mathrm{~mL}$ of $3 \mathrm{M} \mathrm{NaOH}$ and $0.4 \mathrm{~mL}$ of $30 \%$ $\mathrm{H}_{2} \mathrm{O}_{2}$ (dropwise with caution) were added, respectively. The mixture was allowed to stir at ambient temperature for 3 hours. The solution was then quenched with $2 \mathrm{~mL}$ of saturated aqueous $\mathrm{Na}_{2} \mathrm{~S}_{2} \mathrm{O}_{3}$. The two-phase mixture was extracted with ethyl acetate $(2 \times 20 \mathrm{~mL})$ and the combined organic layers were washed with brine $(1 \times 10 \mathrm{~mL})$. The organic layers were then dried over anhydrous $\mathrm{Na}_{2} \mathrm{SO}_{4}$, filtered, and the solvent removed by rotary evaporation. The crude material was purified by silica gel chromatography $(2: 1$ hexanes : ethyl acetate) to provide $19 \mathrm{mg}(68 \%)$ of pure $(S)$-phenyl-ethane-1, 2diol. 
Representative Procedure for Catalytic, Enantioselective Hydrogenation/Homologation/Oxidation Reaction: An oven-dried 2 dram vial equipped with a stirbar was charged with $1.5 \mathrm{mg}(0.004 \mathrm{mmol})$ of bis(norbornadiene)rhodium(I) tetrafluoroborate, $7.4 \mathrm{mg}$ $(0.008 \mathrm{mmol})$ of $(R)$-Walphos (W001), and $1.0 \mathrm{~mL}$ of toluene under an inert atmosphere of argon in a dry-box. The resultant organge-yellow solution was stirred for 10 minutes. After this time, $71 \mathrm{mg}(0.2$ mmol) of cis-1,2-bis(4,4,5,5-tetramethyl-1,3,2-dioxaborolan-2-yl) styrene was added to the solution under argon. The vial was transferred to a Parr hydrogenation bomb. The vessel was charged 3 times to 20 bar with $\mathrm{H}_{2}$ gas. Finally, the vessel was charged to 20 bar, and the reaction was stirred for 24 hours. After this time, volatiles were removed by rotary evaporation. The crude hydrogenation product was dissolved in $0.400 \mathrm{~mL}$ of tetrahydrofuran, and $30 \mu \mathrm{L}(0.44 \mathrm{mmol})$ of bromochloromethane was added. After cooling the solution to $-78^{\circ} \mathrm{C}, 0.28 \mathrm{~mL}(0.44 \mathrm{mmol})$ of $\mathrm{n}$-butyl lithium in hexanes was added dropwise. The mixture was stirred for 10 minutes before warming to room temperature and stirring overnight under an atmosphere of $\mathrm{N}_{2}$. After this time, the reaction mixture was diluted with $1.5 \mathrm{~mL}$ of tetrahydrofuran. $0.8 \mathrm{~mL}$ of $3 \mathrm{M} \mathrm{NaOH}$ and $0.4 \mathrm{~mL}$ of $30 \% \mathrm{H}_{2} \mathrm{O}_{2}$ (dropwise with caution) were added, respectively. The mixture was allowed to stir at ambient temperature for 3 hours. The solution was then quenched with $2 \mathrm{~mL}$ of saturated aqueous $\mathrm{Na}_{2} \mathrm{~S}_{2} \mathrm{O}_{3}$. The two-phase mixture was extracted with ethyl acetate $(2 \times 20 \mathrm{~mL})$ and the combined organic layers were washed with brine $(1 \times 10 \mathrm{~mL})$. The organic layers were then dried over anhydrous $\mathrm{Na}_{2} \mathrm{SO}_{4}$, filtered, and the solvent removed by rotary evaporation. The crude material was purified by silica gel chromatography $(1: 1$ hexanes : ethyl acetate) to provide $28 \mathrm{mg}(86 \%)$ of pure $(S)$-2-phenyl-butane-1,4-diol.

Representative Synthesis of Vinyl 1,2-Bis(boronates): An oven-dried $20 \mathrm{~mL}$ vial equipped with a stir-bar was charged with $187 \mathrm{mg}(0.15 \mathrm{mmol})$ of tetrakis(triphenylphosphine)platinum (0) and $762 \mathrm{mg}$ (3.0 mmol) of bis(pinacolato)diboron under an inert atmosphere of argon in a dry-box. The solids were dissolved in $10 \mathrm{~mL}$ of anhydrous dimethylformamide. The corresponding alkyne (3.6 mmol) was transferred to the vial via syringe. The vial was sealed and heated to $100{ }^{\circ} \mathrm{C}$ for $24-96$ hours. After this time, the mixture was cooled to room temperature and transferred to a separatory funnel containing 50 $\mathrm{mL}$ of diethyl ether. The organic layer was washed with water $(2 \times 10 \mathrm{~mL})$ and once with $10 \mathrm{~mL}$ of brine. The organics were dried over anhydrous $\mathrm{Na}_{2} \mathrm{SO}_{4}$, filtered, and the solvent was removed by rotary evaporation. The resulting oil was distilled under vacuum.
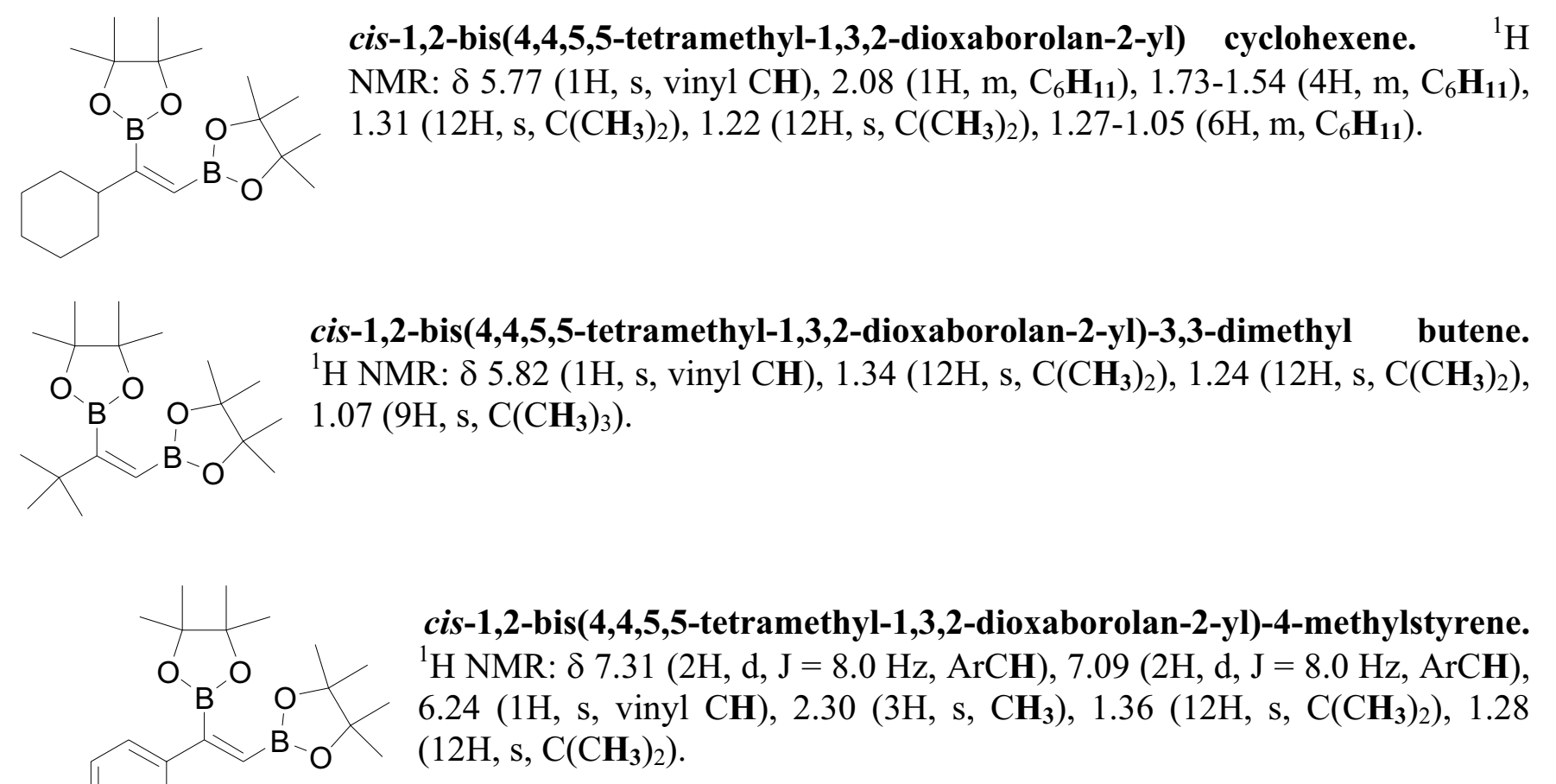

$\mathrm{Me}$

Page S - 3 


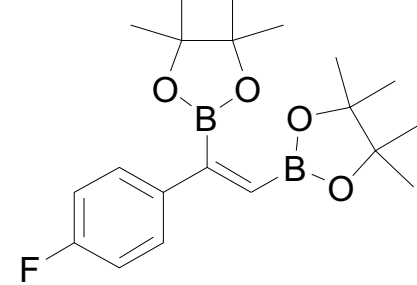

cis-1,2-bis(4,4,5,5-tetramethyl-1,3,2-dioxaborolan-2-yl)-4-fluorostyrene. ${ }^{1} \mathrm{H}$ NMR: $\delta 7.38(2 \mathrm{H}, \mathrm{dd}, \mathrm{J}=8.8 \mathrm{~Hz}, 5.6 \mathrm{~Hz}, \operatorname{ArCH}), 6.96(2 \mathrm{H}, \mathrm{t}, \mathrm{J}=8.8 \mathrm{~Hz}$, $\operatorname{ArCH}), 6.22(1 \mathrm{H}, \mathrm{s}$, vinyl $\mathrm{CH}), 1.35\left(12 \mathrm{H}, \mathrm{s}, \mathrm{C}\left(\mathrm{CH}_{3}\right)_{2}\right), 1.29(12 \mathrm{H}, \mathrm{s}$, $\left.\mathrm{C}\left(\mathrm{CH}_{3}\right)_{2}\right)$.

OH (S)-1-cyclohexyl-ethane-1,2-diol. IR (neat): 3448 (br, s) cm ${ }^{-1} ;{ }^{1} \mathrm{H}$ NMR: $\delta 3.68(1 \mathrm{H}$, $\mathrm{OH} \mathrm{dd}, \mathrm{J}=10.4 \mathrm{~Hz}, 2.0 \mathrm{~Hz}, \mathrm{CHCHOH}), 3.51\left(1 \mathrm{H}, \mathrm{m}, \mathrm{CH}_{2} \mathrm{OH}\right), 3.42\left(1 \mathrm{H}, \mathrm{m}, \mathrm{CH}_{2} \mathrm{OH}\right)$, $2.09\left(2 \mathrm{H}\right.$, broad s, OH), $1.84\left(1 \mathrm{H}, \mathrm{d}, \mathrm{J}=12.8 \mathrm{~Hz}, \mathrm{C}_{6} \mathbf{H}_{11}\right), 1.73\left(2 \mathrm{H}, \mathrm{m}, \mathrm{C}_{6} \mathbf{H}_{11}\right), 1.63$ $\left(2 \mathrm{H}, \mathrm{m}, \mathrm{C}_{6} \mathbf{H}_{11}\right), 0.9-1.5\left(6 \mathrm{H}, \mathrm{m}, \mathrm{C}_{6} \mathbf{H}_{11}\right) ;{ }^{13} \mathrm{C}$ NMR: $\delta$ 76.5, 64.8, 40.7, 28.9, 28.6, 26.3, 26.0, 25.9. HRMS (FAB) Calc'd for $\mathrm{C}_{8} \mathrm{H}_{16} \mathrm{O}_{2}\left(\mathrm{M}+\mathrm{NH}_{4}\right)^{+}:$162.1489. Found $\left(\mathrm{M}+\mathrm{NH}_{4}\right)^{+}: 162.1500$.

Proof of Stereochemistry. Stereochemical ratios were determined in comparison to authentic racemic materials prepared by osmium tetraoxide dihydroxylation. Absolute stereochemistry established in comparison to authentic $(R)$ isomer prepared via a Sharpless asymmetric dihydroxylation (Becker, H.; King, S. B.; Taniguchi, M.; Vanhessche, K.; Sharpless, K. B. J. Org. Chem. 1995, 60, 3940).

Chiral GLC ( $\beta$-dex, Supelco, $130^{\circ} \mathrm{C}$ ) analysis of the acetonide (dimethoxypropane/cat. pTsOH) product:

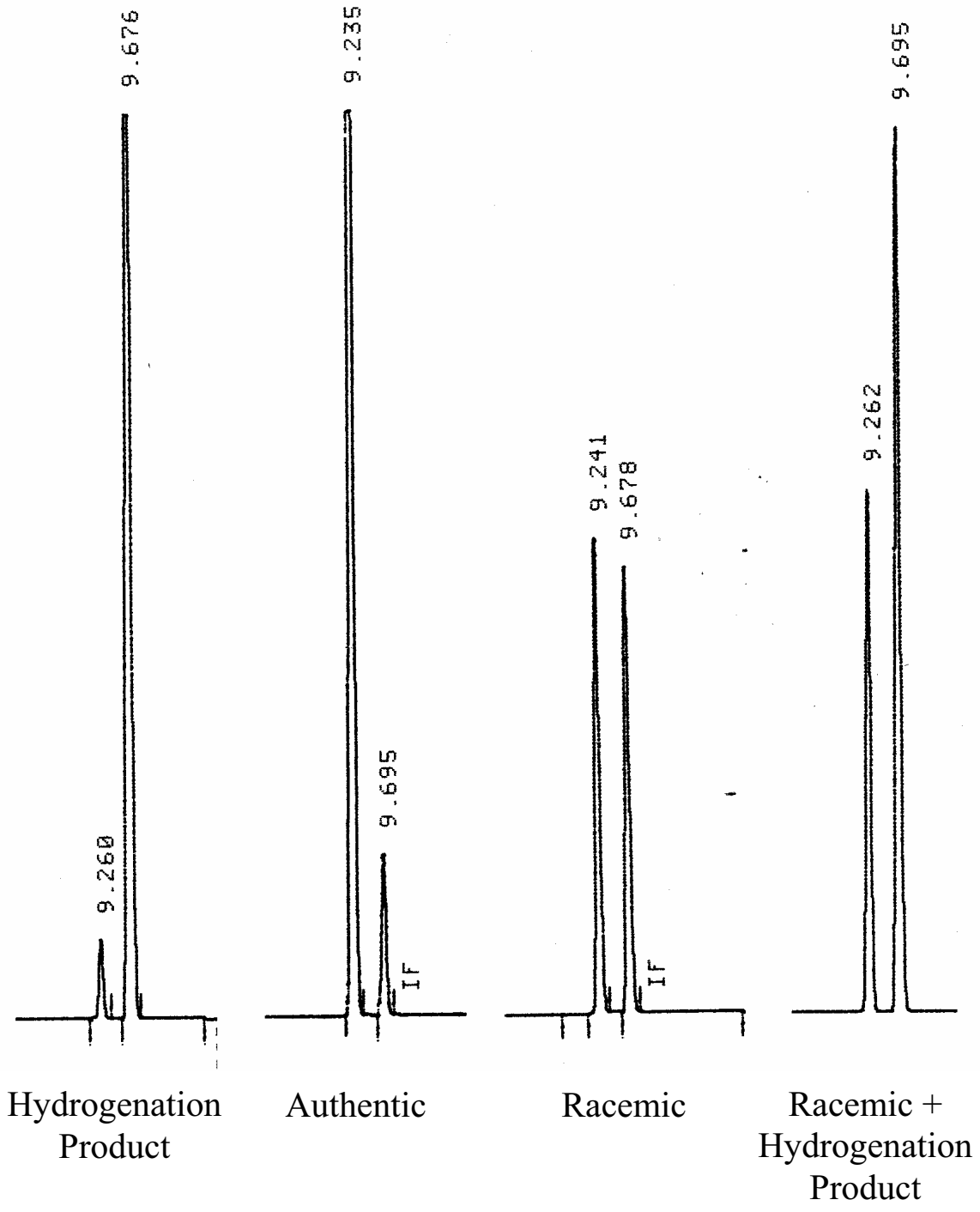

Page S - 4 
$\mathrm{OH} \quad(\boldsymbol{S})$-heptane-1,2-diol. IR (neat): 3460 (br, s) $\mathrm{cm}^{-1} ;{ }^{1} \mathrm{H}$ NMR: $\delta 3.68(1 \mathrm{H}, \mathrm{m}$, $\left.\mathrm{OH} \mathrm{CH}_{2} \mathrm{CHOH}\right), 3.63\left(1 \mathrm{H}, \mathrm{d}, \mathrm{J}=10.8 \mathrm{~Hz}, 2.8 \mathrm{~Hz}, \mathrm{CH}_{\mathrm{a}} \mathrm{H}_{\mathrm{b}} \mathrm{OH}\right), 3.41(1 \mathrm{H}, \mathrm{dd}, \mathrm{J}=10.8$ $\left.\mathrm{Hz}, 7.6 \mathrm{~Hz}, \mathrm{CH}_{\mathrm{a}} \mathbf{H}_{\mathbf{b}} \mathrm{OH}\right), 2.29$ (2H, br s, OH), 1.5-1.2 (8H, m, -CH $\left.\mathbf{2}^{-}\right), 0.87$ (3H, t, $\left.\mathrm{J}=6.4 \mathrm{~Hz}, \mathrm{CH}_{3}\right) ;{ }^{13} \mathrm{C}$ NMR: $\delta$ 72.3, 66.8, 33.1, 31.8, 25.2, 22.5, 29.2, 13.9. HRMS (FAB) Calc'd for $\mathrm{C}_{7} \mathrm{H}_{16} \mathrm{O}_{2}\left(\mathrm{M}+\mathrm{NH}_{4}\right)^{+}: 150.1489$. Found $\left(\mathrm{M}+\mathrm{NH}_{4}\right)^{+}: 150.1499$.

Proof of Stereochemistry. Stereochemical ratios were determined in comparison to authentic racemic materials prepared by osmium tetraoxide dihydroxylation. Absolute stereochemistry established in comparison to authentic $(R)$ isomer prepared via a Sharpless asymmetric dihydroxylation (Becker, H.; King, S. B.; Taniguchi, M.; Vanhessche, K.; Sharpless, K. B. J. Org. Chem. 1995, 60, 3940).

Chiral GLC ( $\beta$-dex, Supelco, $100{ }^{\circ} \mathrm{C}$ ) analysis of the acetonide (dimethoxypropane/cat. pTsOH) product:

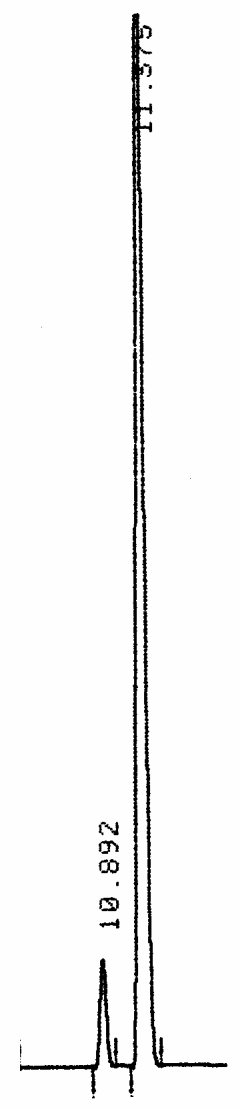

Hydrogenation

Product

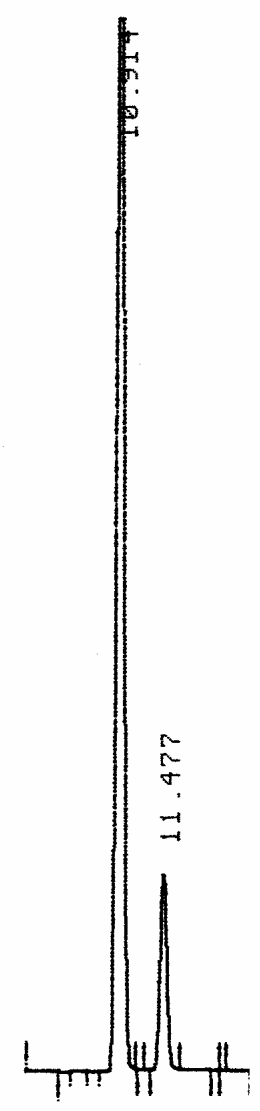

Authentic

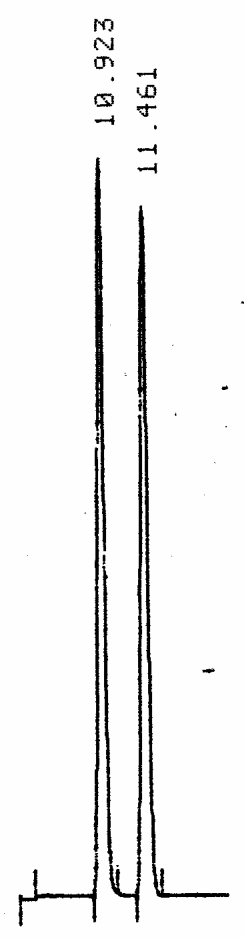

Racemic

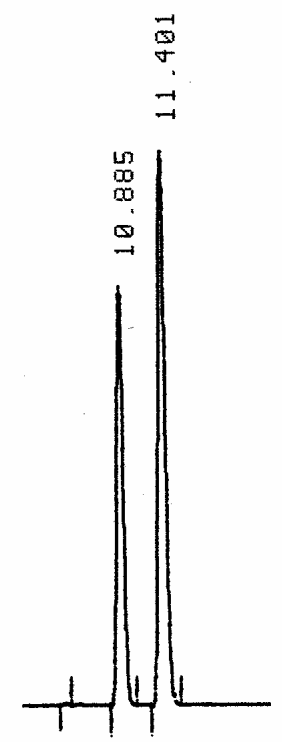

Racemic + Hydrogenation

Product 
$\mathrm{OH}$

(S)-3,3-dimethyl-butane-1,2-diol. IR (neat): 3450 (br, s) cm ${ }^{-1} ;{ }^{1} \mathrm{H}$ NMR: $\delta 3.70(1 \mathrm{H}$, dd, $\mathrm{OH} \mathrm{J}=10.4 \mathrm{~Hz}, 2.0 \mathrm{~Hz}, \mathrm{CHOH}), 3.45\left(1 \mathrm{H}, \mathrm{t}, \mathrm{J}=10.4 \mathrm{~Hz}, \mathrm{CH}_{\mathbf{a}} \mathrm{H}_{\mathrm{b}} \mathrm{OH}\right), 3.34(1 \mathrm{H}, \mathrm{dd}, \mathrm{J}=9.2$ $\left.\mathrm{Hz}, 2.0 \mathrm{~Hz}, \mathrm{CH}_{\mathrm{a}} \mathbf{H}_{\mathbf{b}} \mathrm{OH}\right), 2.63\left(2 \mathrm{H}\right.$, br s, OH), $0.88\left(9 \mathrm{H}, \mathrm{s}, \mathrm{C}\left(\mathrm{CH}_{3}\right)_{3}\right) ;{ }^{13} \mathrm{C} \mathrm{NMR}: \delta 79.7$, 63.1, 33.5, 25.8. HRMS (FAB) Calc'd for $\mathrm{C}_{6} \mathrm{H}_{14} \mathrm{O}_{2}\left(\mathrm{M}+\mathrm{NH}_{4}\right)^{+}$: 136.1332 Found $\left(\mathrm{M}+\mathrm{NH}_{4}\right)^{+}$: 136.1341.

Proof of Stereochemistry. Stereochemical ratios were determined in comparison to authentic racemic materials prepared by osmium tetraoxide dihydroxylation. Absolute stereochemistry established in comparison to authentic $(R)$ isomer prepared via a Sharpless asymmetric dihydroxylation (Becker, H.; King, S. B.; Taniguchi, M.; Vanhessche, K.; Sharpless, K. B. J. Org. Chem. 1995, 60, 3940).

Chiral GLC ( $\beta$-dex, Supelco, $\left.120^{\circ} \mathrm{C}\right)$ analysis of diol product:

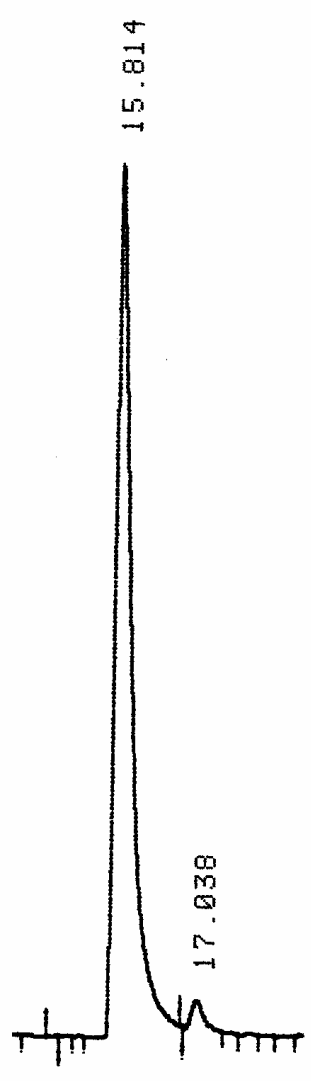

Hydrogenation

Product

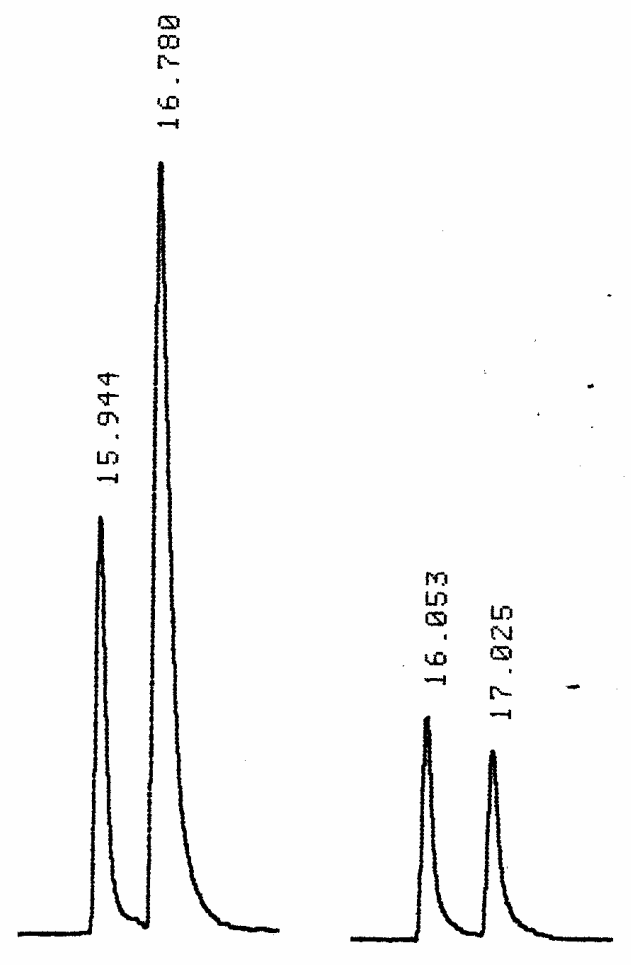

Authentic
Racemic

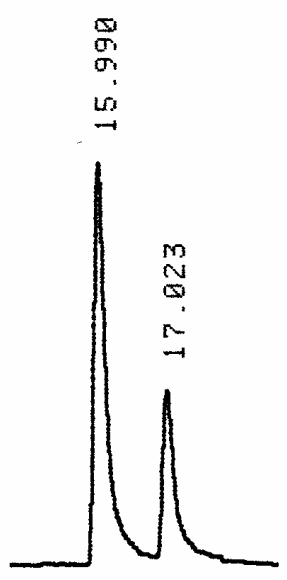

Racemic + Hydrogenation Product 
$\mathrm{OH}$

(S)-phenyl-ethane-1, 2-diol. IR (neat): 3438 (br, s) $\mathrm{cm}^{-1} ;{ }^{1} \mathrm{H}$ NMR: $\delta$ 7.4-7.26 (5H, m,

$\mathrm{OH} \mathrm{Ph}), 4.80(1 \mathrm{H}, \mathrm{dd}, \mathrm{J}=8.0 \mathrm{~Hz}, 3.2 \mathrm{~Hz}, \mathrm{PhCHOH}), 3.73(1 \mathrm{H}, \mathrm{dd}, \mathrm{J}=10.8 \mathrm{~Hz}, 3.2 \mathrm{~Hz}$, $\left.\mathrm{CH}_{\mathbf{a}} \mathrm{H}_{\mathrm{b}} \mathrm{OH}\right), 3.64\left(1 \mathrm{H}, \mathrm{dd}, \mathrm{J}=11.2 \mathrm{~Hz}, 8.0 \mathrm{~Hz}, \mathrm{CH}_{\mathrm{a}} \mathbf{H}_{\mathbf{b}} \mathrm{OH}\right), 2.75(2 \mathrm{H}$, br s, OH), 2.32 $\left(2 \mathrm{H}\right.$, br s, OH); ${ }^{13} \mathrm{C}$ NMR: $\delta 140.5,128.5,128.0,126.0,74.7,68.1$. HRMS (FAB) Calc'd for $\mathrm{C}_{8} \mathrm{H}_{10} \mathrm{O}_{2}\left(\mathrm{M}+\mathrm{NH}_{4}\right)^{+}:$156.1019 Found $\left(\mathrm{M}+\mathrm{NH}_{4}\right)^{+}: 156.1026$.

Proof of Stereochemistry. Stereochemical ratios were determined in comparison to authentic racemic materials prepared by osmium tetraoxide dihydroxylation. Absolute stereochemistry established in comparison to authentic $(R)$ isomer prepared via a Sharpless asymmetric dihydroxylation (Becker, H.; King, S. B.; Taniguchi, M.; Vanhessche, K.; Sharpless, K. B. J. Org. Chem. 1995, 60, 3940).

Chiral GLC ( $\beta$-dex, Supelco, $140{ }^{\circ} \mathrm{C}$ ) analysis of the acetonide (dimethoxypropane/cat. $p T_{s} O H$ ) product:

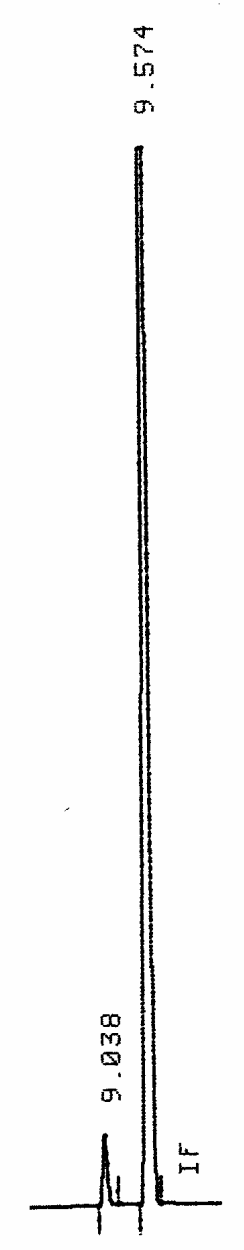

Hydrogenation

Product

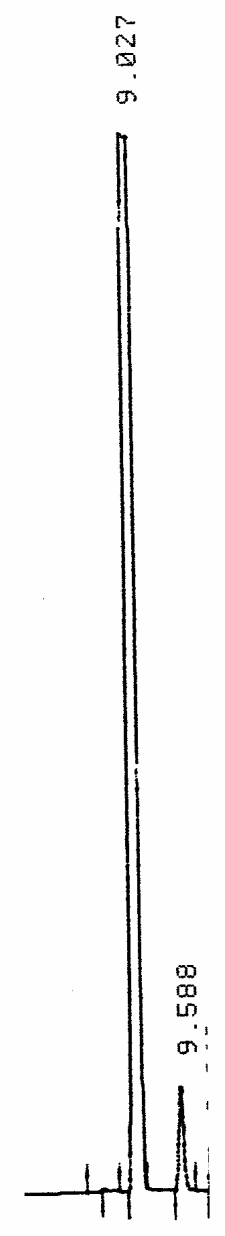

Authentic

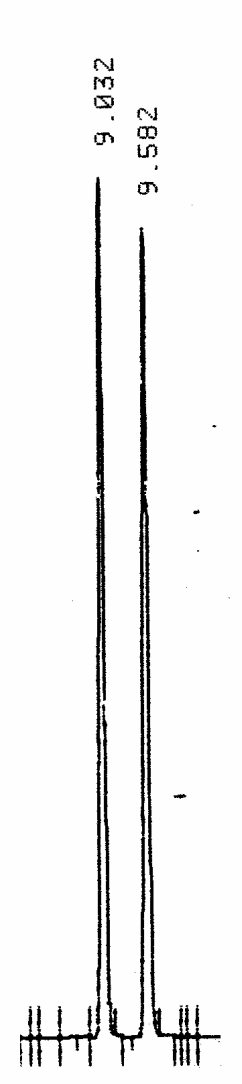

Racemic $\stackrel{n}{n}$

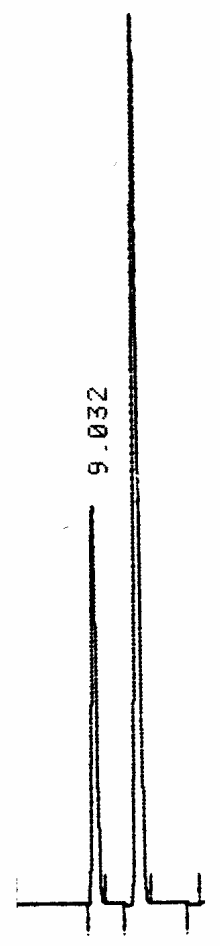

Racemic + Hydrogenation

Product 
(S)-1-(4-fluoro-phenyl)-ethane-1,2-diol. IR (neat): 3467 (br, s) $\mathrm{cm}^{-1} ;{ }^{1} \mathrm{H}$ NMR: $\delta$

$\mathrm{OH} 7.30(2 \mathrm{H}, \mathrm{dd}),, 7.02(2 \mathrm{H}, \mathrm{t}),, 4.77(1 \mathrm{H}, \mathrm{dd}, \mathrm{J}=8.4 \mathrm{~Hz}, 3.2 \mathrm{~Hz}, \mathrm{CHOH}), 3.70(1 \mathrm{H}$, $\left.\mathrm{dd}, \mathrm{J}=11.2 \mathrm{~Hz}, 3.2 \mathrm{~Hz}, \mathrm{CH}_{\mathbf{a}} \mathrm{H}_{\mathrm{b}} \mathrm{OH}\right), 3.59\left(1 \mathrm{H}, \mathrm{dd}, \mathrm{J}=11.2 \mathrm{~Hz}, 8.4 \mathrm{~Hz}, \mathrm{CH}_{\mathrm{a}} \mathbf{H}_{\mathbf{b}} \mathrm{OH}\right)$, $2.95\left(1 \mathrm{H}\right.$, br s, OH), $2.47(1 \mathrm{H}$, br s, OH $) ;{ }^{13} \mathrm{C}$ NMR: $\delta 162.4(\mathrm{~d}, \mathrm{~J}=244.5 \mathrm{~Hz})$, $136.2(\mathrm{~d}, \mathrm{~J}=3.0 \mathrm{~Hz}), 127.7(\mathrm{~d}, \mathrm{~J}=8.0 \mathrm{~Hz}), 115.4(\mathrm{~d}, \mathrm{~J}=21.3 \mathrm{~Hz})$, 74.0, 68.0. HRMS (FAB) Calc'd for $\mathrm{C}_{8} \mathrm{H}_{9} \mathrm{O}_{2} \mathrm{~F}\left(\mathrm{M}+\mathrm{NH}_{4}\right)^{+}: 174.0925$ Found $\left(\mathrm{M}+\mathrm{NH}_{4}\right)^{+}: 174.0928$.

Proof of Stereochemistry. Stereochemical ratios were determined in comparison to authentic racemic materials prepared by osmium tetraoxide dihydroxylation. Absolute stereochemistry established in comparison to authentic $(R)$ isomer (assignment made based on Sharpless stereochemical model) prepared via a Sharpless asymmetric dihydroxylation.

Chiral GLC ( $\beta$-dex, Supelco, $140{ }^{\circ} \mathrm{C}$ ) analysis of the acetonide (dimethoxypropane/cat. pTsOH) product:

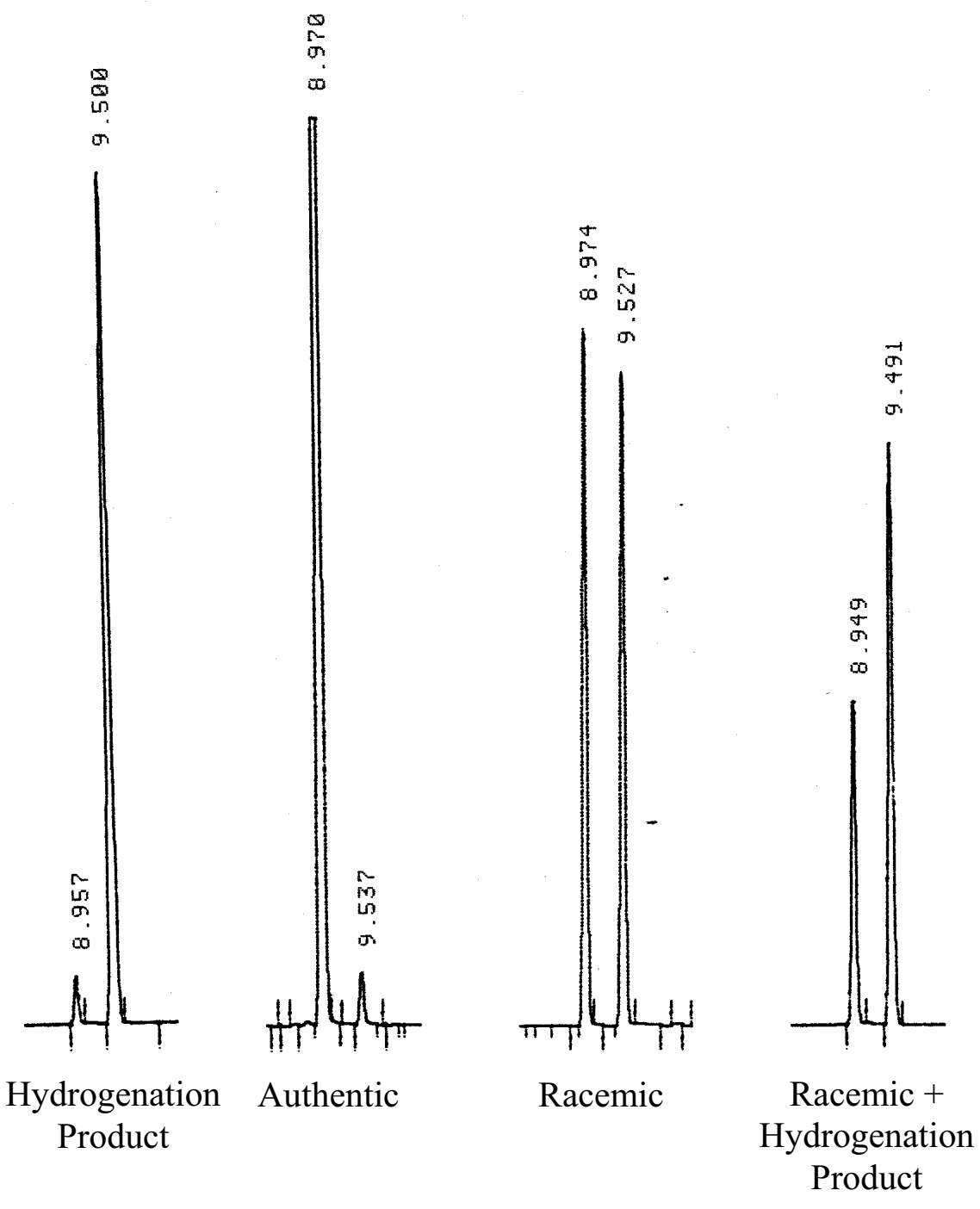

Page S - 8 
(S)-1-p-tolyl-ethane-1,2-diol. IR (neat): 3465 (br, s) cm ${ }^{-1} ;{ }^{1} \mathrm{H}$ NMR: $\delta 7.22(2 \mathrm{H}$, $\mathrm{OH} \mathrm{d}, \mathrm{J}=8.0 \mathrm{~Hz}, \operatorname{ArH}), 7.14(2 \mathrm{H}, \mathrm{d}, \mathrm{J}=8.0 \mathrm{~Hz}, \operatorname{ArH}), 4.75(1 \mathrm{H}, \mathrm{dd}, \mathrm{J}=8.0 \mathrm{~Hz}, 3.2$ $\mathrm{Hz}, \mathrm{CHOH}), 3.70\left(1 \mathrm{H}, \mathrm{dd}, \mathrm{J}=11.2 \mathrm{~Hz}, 3.2 \mathrm{~Hz}, \mathrm{CH}_{\mathrm{a}} \mathrm{H}_{\mathrm{b}} \mathrm{OH}\right), 3.62(1 \mathrm{H}, \mathrm{dd}, \mathrm{J}=11.2$ $\mathrm{Me}$ $\left.\mathrm{Hz}, 8.0 \mathrm{~Hz}, \mathrm{CH}_{\mathrm{a}} \mathbf{H}_{\mathrm{b}} \mathrm{OH}\right), 2.74(1 \mathrm{H}$, br s, OH), $2.37(1 \mathrm{H}$, br s, OH), $2.32(3 \mathrm{H}, \mathrm{s}$, $\left.\mathrm{CH}_{3}\right) ;{ }^{13} \mathrm{C}$ NMR: $\delta 137.7,137.5,129.2,126.0,74.5,68.0,21.1$. HRMS (FAB) Calc'd for $\mathrm{C}_{9} \mathrm{H}_{12} \mathrm{O}_{2}(\mathrm{M}+$ $\left.\mathrm{NH}_{4}\right)^{+}: 170.1176$ Found $\left(\mathrm{M}+\mathrm{NH}_{4}\right)^{+}: 170.1196$.

Proof of Stereochemistry. Stereochemical ratios were determined in comparison to authentic racemic materials prepared by osmium tetraoxide dihydroxylation. Absolute stereochemistry established in comparison to authentic $(R)$ isomer (assignment made based on Sharpless stereochemical model) prepared via a Sharpless asymmetric dihydroxylation.

Chiral GLC ( $\beta$-dex, Supelco, $140{ }^{\circ} \mathrm{C}$ ) analysis of the acetonide (dimethoxypropane/cat. $p T_{s} O H$ ) product:

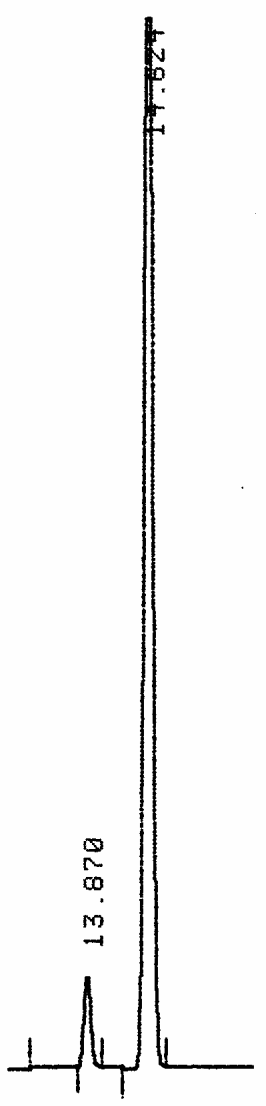

Hydrogenation

Product

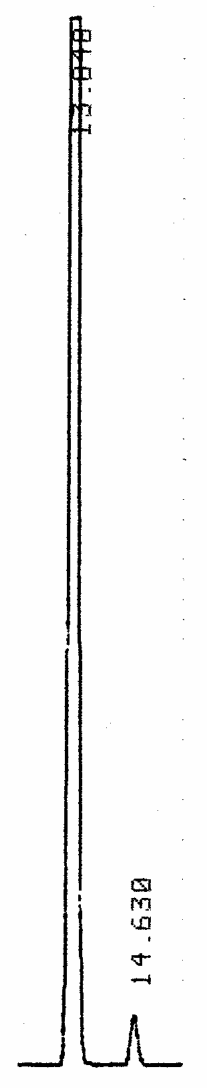

Authentic

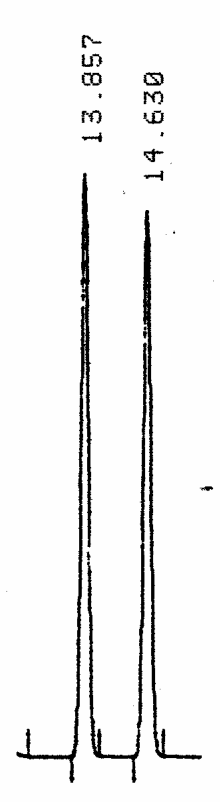

Racemic

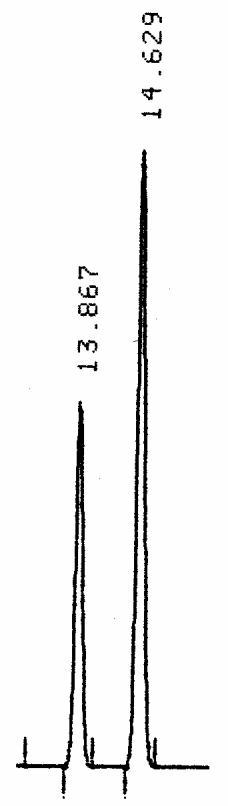

Racemic + Hydrogenation Product 
(S)-2-phenyl-butane-1,4-diol. IR (neat): 3460 (br s) $\mathrm{cm}^{-1} ;{ }^{1} \mathrm{H}$ NMR: $\delta$ 7.33-7.28 $\left(2 \mathrm{H}, \mathrm{m}\right.$, aromatic), 7.24-7.18 (3H, m, aromatic), $3.74\left(2 \mathrm{H}, \mathrm{d}, \mathrm{J}=6.4 \mathrm{~Hz}, \mathrm{CH}_{2} \mathrm{OH}\right)$, $\mathrm{OH} 3.66\left(1 \mathrm{H}, \mathrm{m}, \mathrm{CH}_{2} \mathrm{OH}\right), 3.54\left(1 \mathrm{H}, \mathrm{ddd}, \mathrm{J}=10.8 \mathrm{~Hz}, \mathrm{~J}=8.0 \mathrm{~Hz}, \mathrm{~J}=5.2 \mathrm{~Hz}, \mathrm{CH}_{2} \mathrm{OH}\right)$, $2.93\left(1 \mathrm{H}\right.$, pent, J = 6.8 Hz, CHAr), $2.36(2 \mathrm{H}$, br s, $\mathrm{OH}), 2.00\left(1 \mathrm{H}, \mathrm{m}, \mathrm{CH}_{2} \mathrm{CH}_{2} \mathrm{OH}\right)$, $1.86\left(1 \mathrm{H}, \mathrm{m}, \mathrm{CH}_{2} \mathrm{CH}_{2} \mathrm{OH}\right) ;{ }^{13} \mathrm{C}$ NMR: $\delta 142.3,128.7,127.8,126.8,67.5,61.0,45.9,35.8$. HRMS (FAB) Calc'd for $\mathrm{C}_{10} \mathrm{H}_{14} \mathrm{O}_{2}\left(\mathrm{M}+\mathrm{NH}_{4}\right)^{+}: 184.1332$ Found $\left(\mathrm{M}+\mathrm{NH}_{4}\right)^{+}: 184.1342$.

Proof of Stereochemistry. Stereochemical ratios were determined in comparison to authentic racemic material prepared by homologation of racemic 1,2-bis(boronate). Absolute stereochemistry established by analogy to the corresponding diol product.

Chiral SFC (AD-H, Chiracel, 4.0\% Methanol, $3.0 \mathrm{~mL} / \mathrm{min}$ ) analysis of alcohol product:

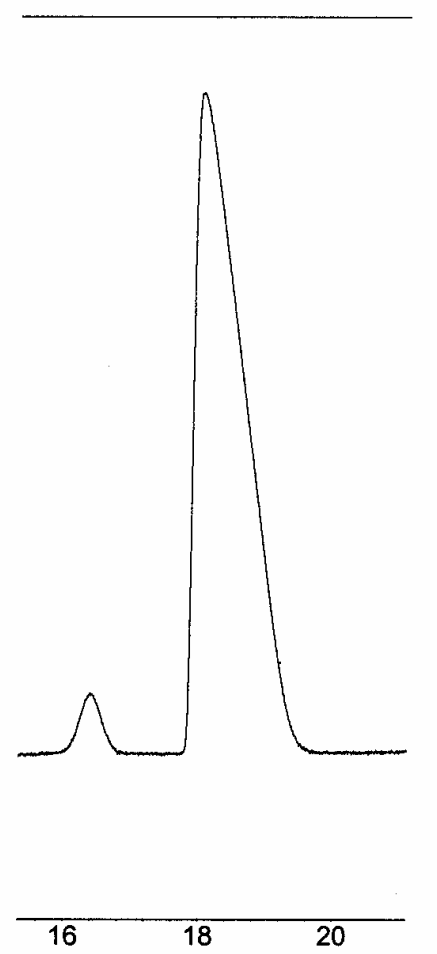

Hydrogenation/ Homologation

Product

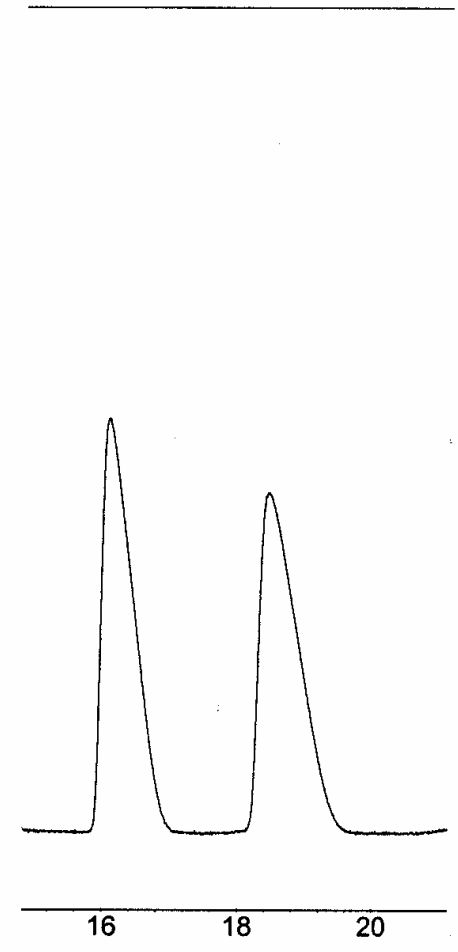

Racemic

Racemic + Hydrogenation/ Homologation

Product 

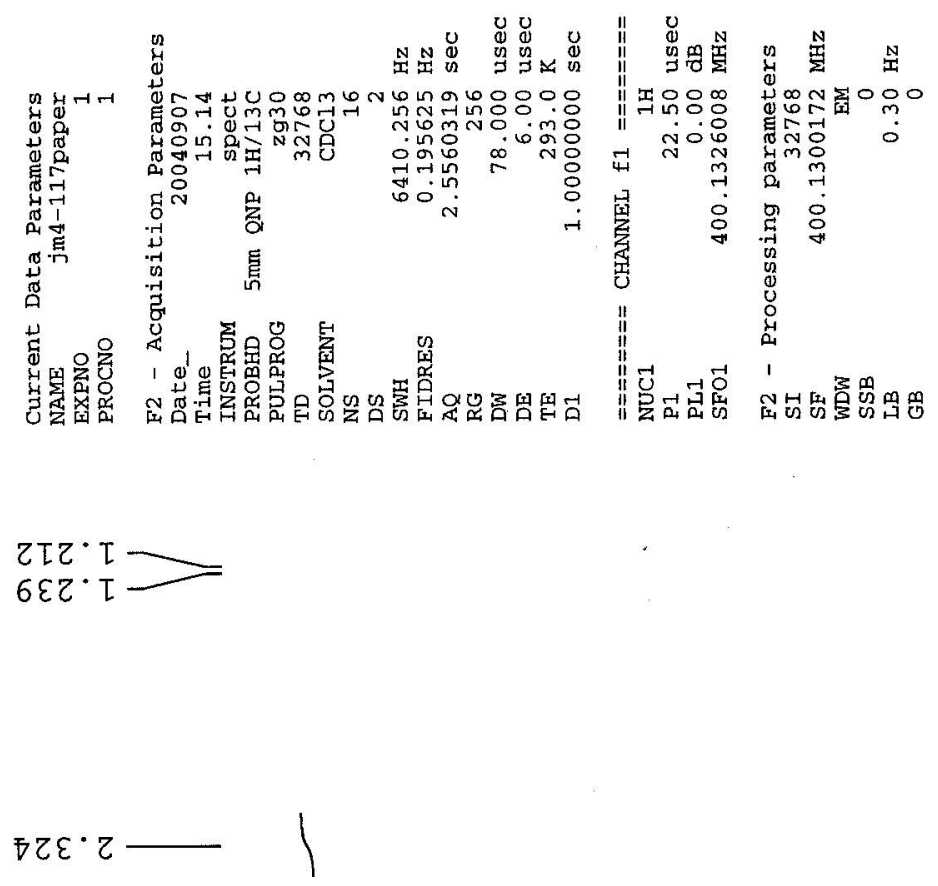

$8 S L \cdot z-1$
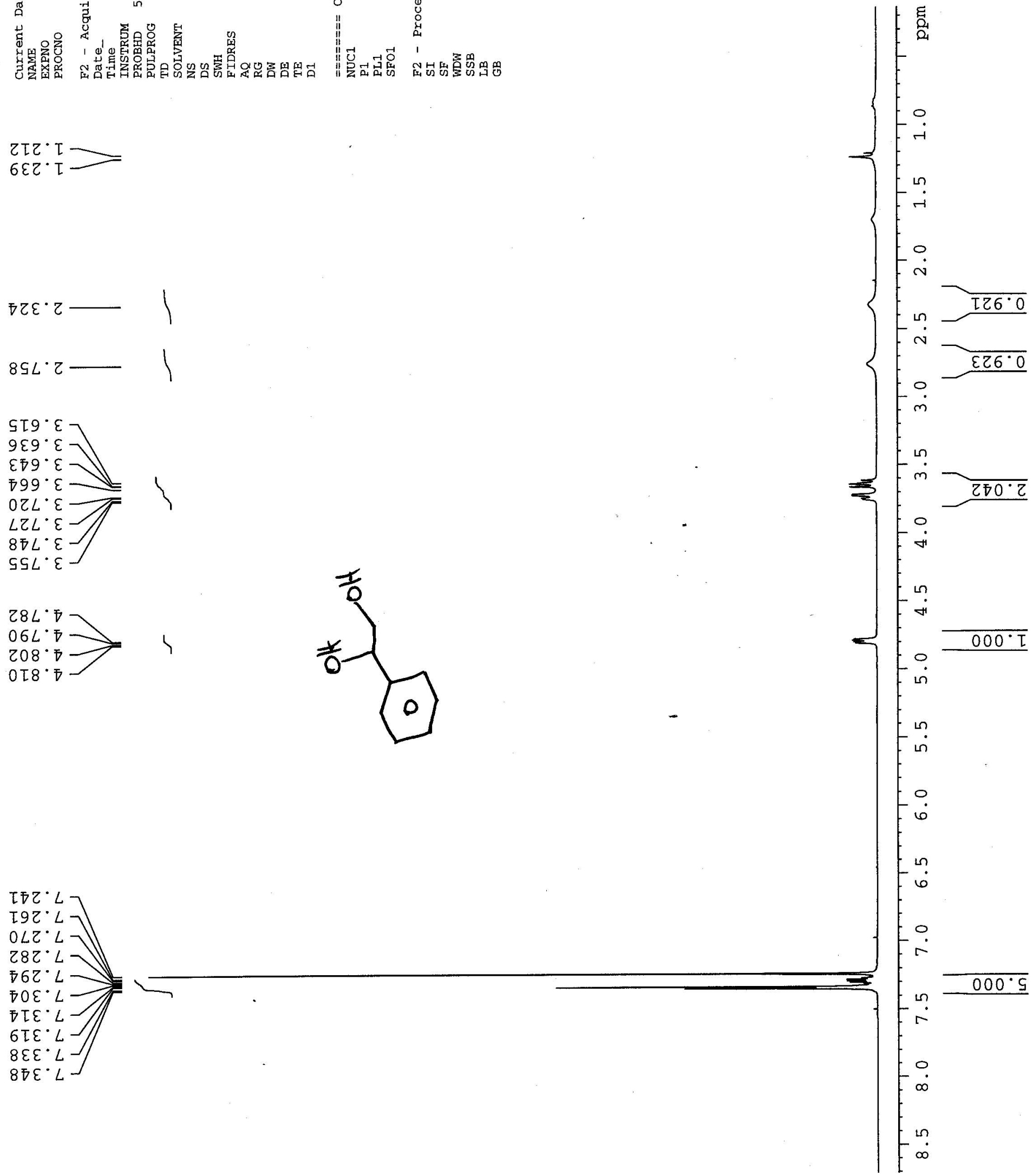

Page S - 11 

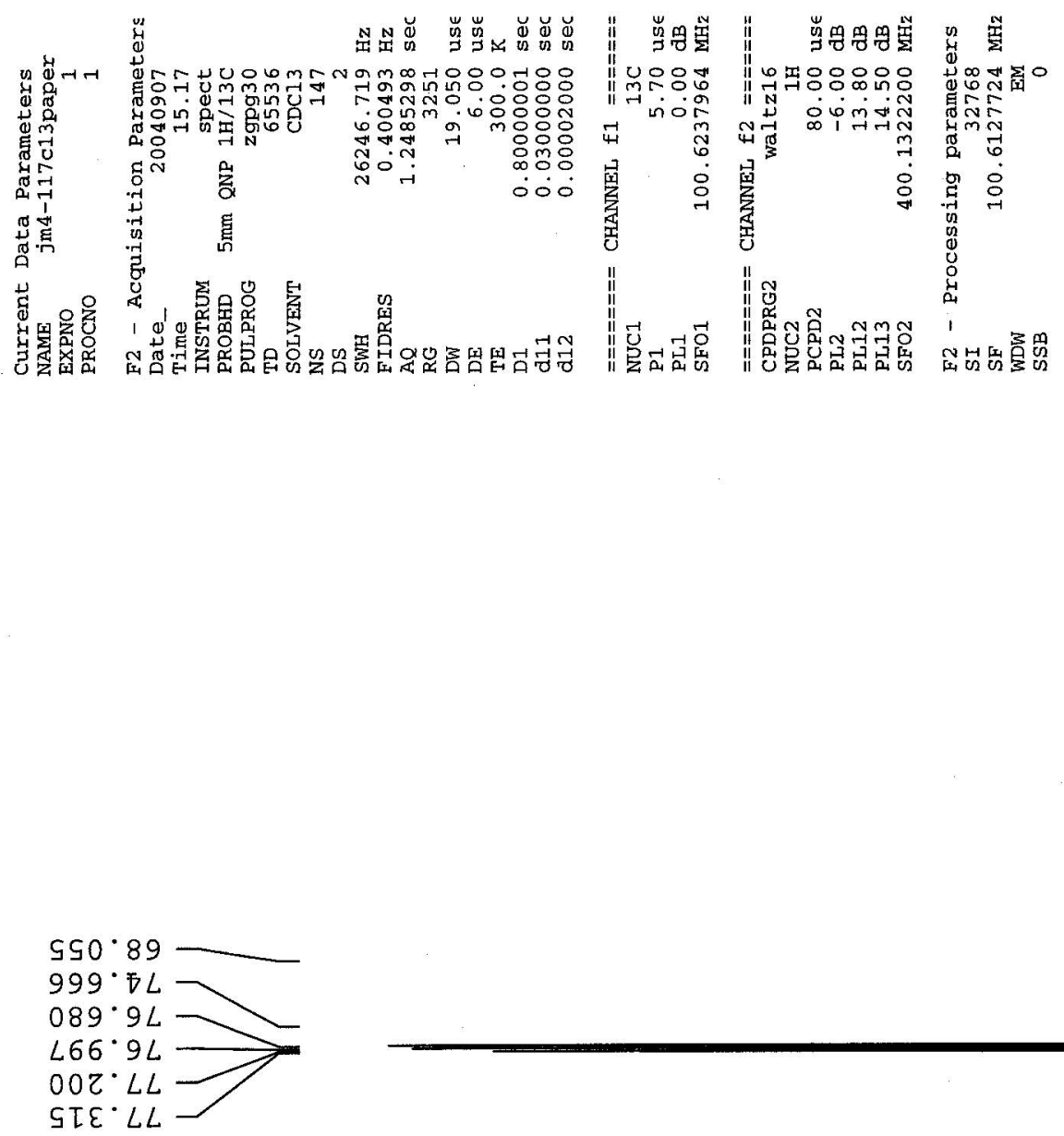

$6 \varepsilon 0 \cdot 9 \tau \tau$ $666^{\circ} \mathrm{LZT}$ $8 Z 5 \cdot 8 Z \mathrm{~T}$

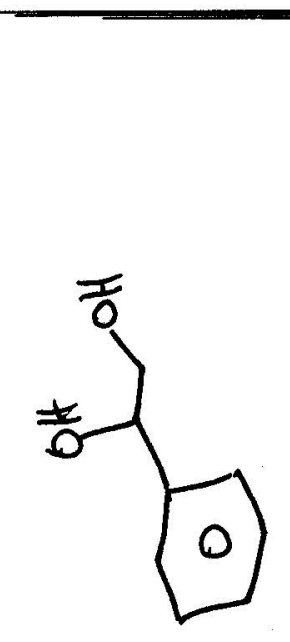

097.0DT
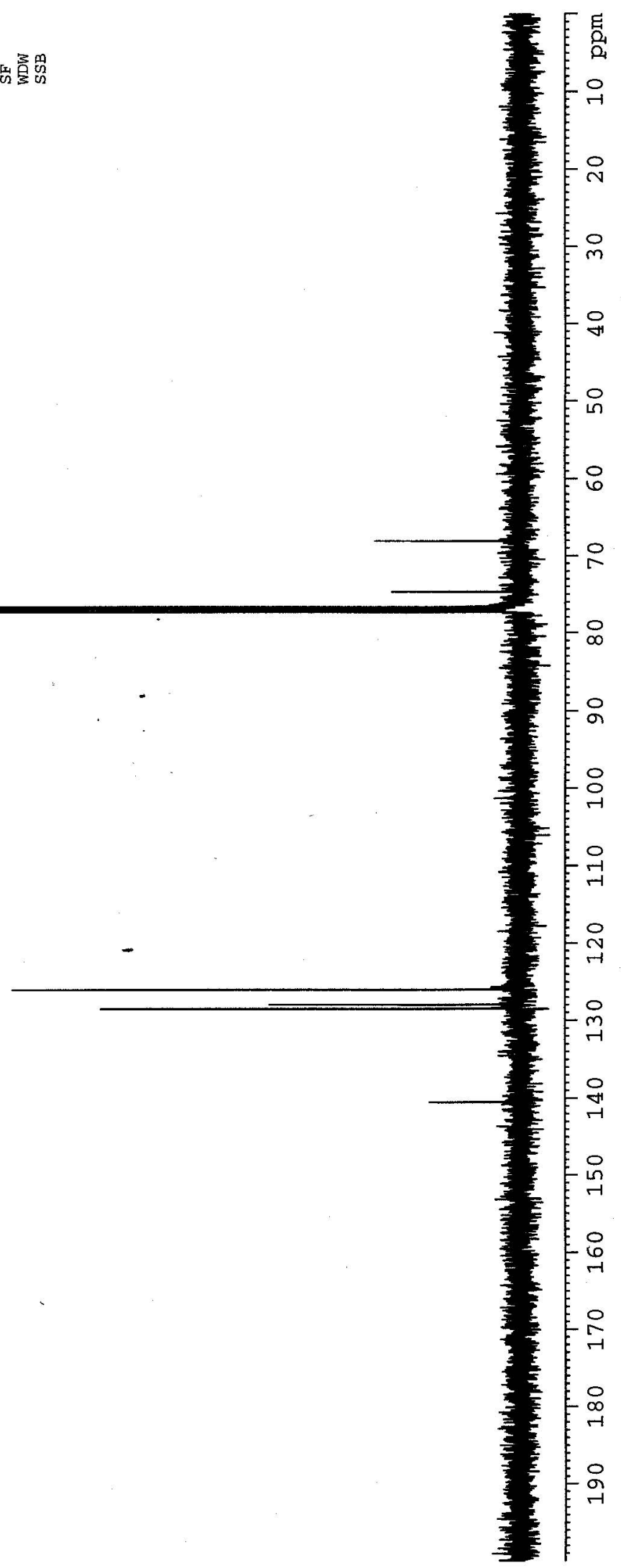

Page S - 12 

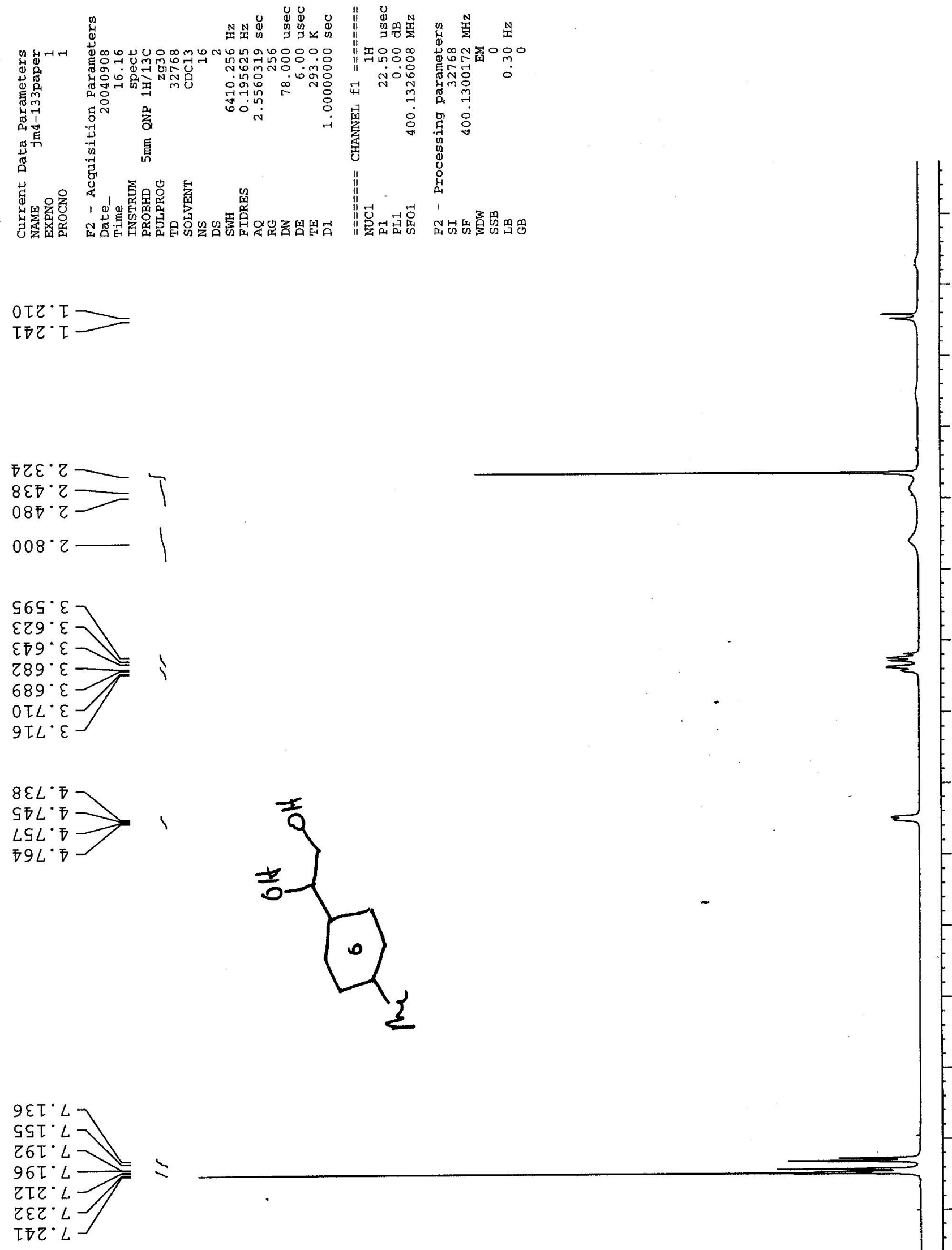

$-1$

ㄴำ

웅

$\therefore$

$\frac{270^{\circ} \varepsilon}{200^{\circ}}$

$696^{\circ} 0$

$m$

$E$ ?

$\frac{\tau=\frac{\tau Z 0^{\circ} I}{}}{670^{\circ} \tau}$

?

$+$

4

${ }_{0}=\underline{000 \cdot \tau}$

\%

.

?

-

$-0$

6

$-\stackrel{4}{9}$

6

0

$\therefore \frac{\sqrt{090^{\circ} z}}{\underline{607^{\circ} z}}$

$-?$

Er

$-0$

$-\infty$

In

$-\infty$

Page S - 13 


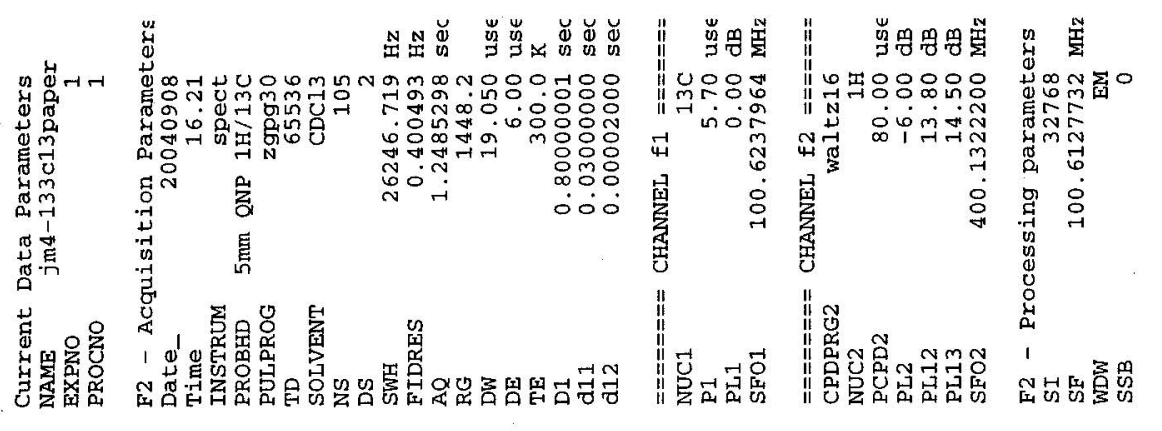

$\varsigma 60^{\circ} \tau Z$
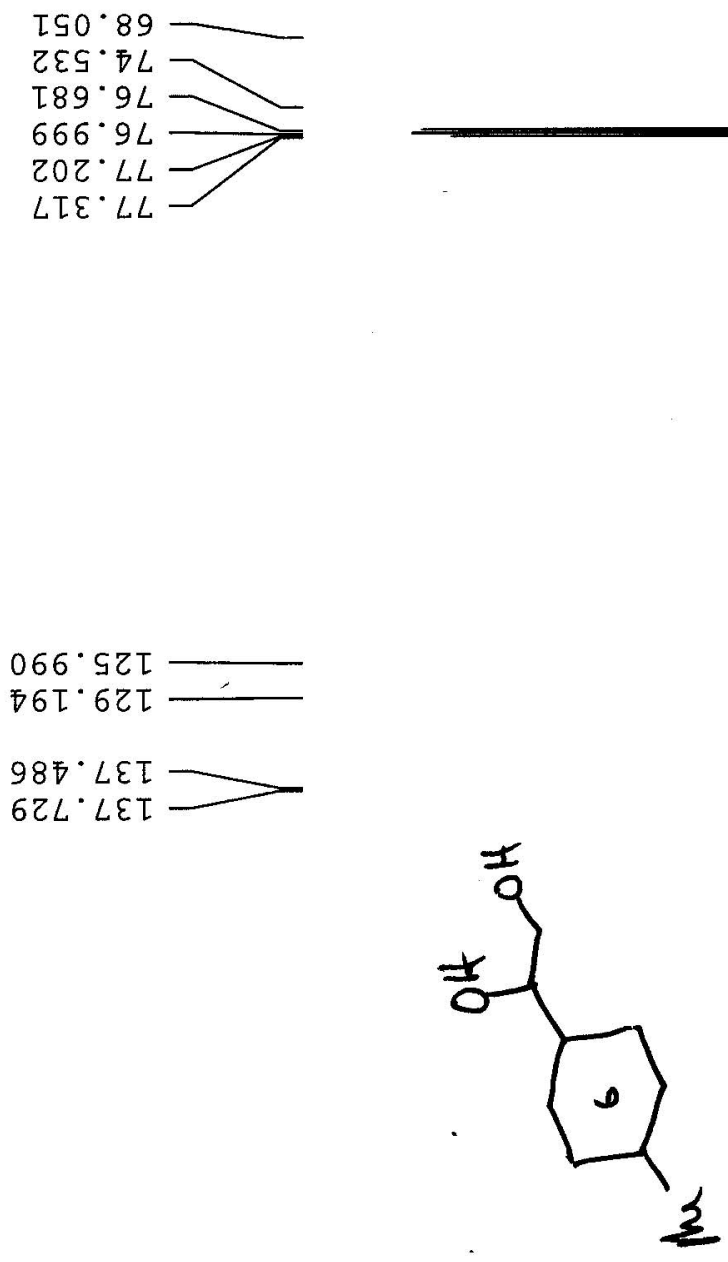

Page S - 14 


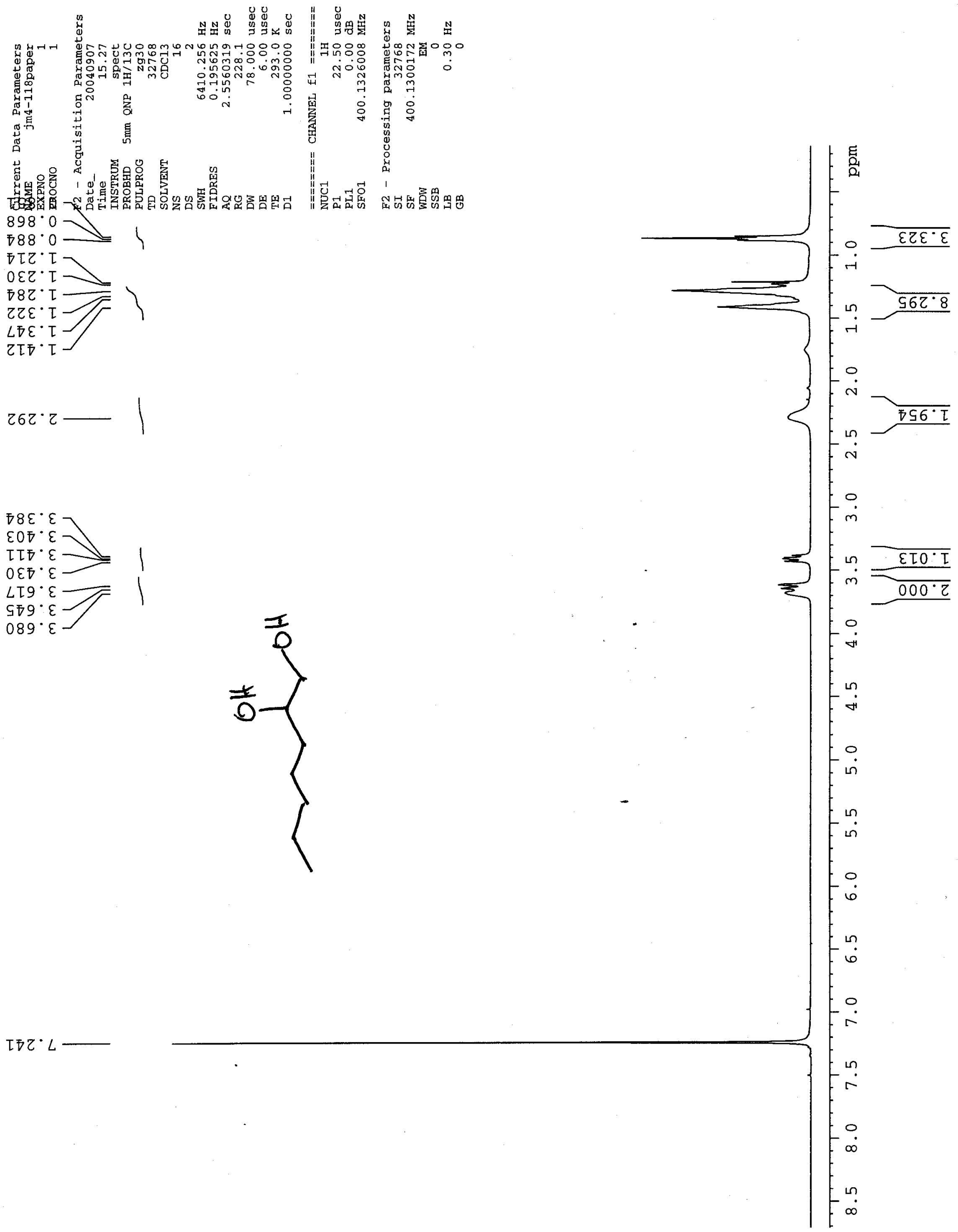

Page S - 15 


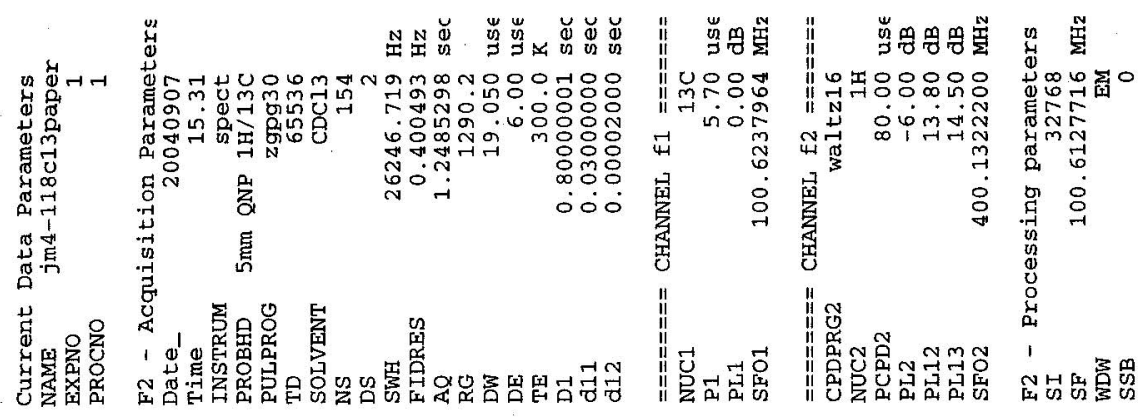

$8 L \sigma^{\circ} \varepsilon \tau$

$\angle \varepsilon S^{\circ} \mathrm{zz}$

$06 \tau$ ५ $\mathrm{Z}$

$\varepsilon 08^{\circ} \tau \varepsilon$

$₹ \tau \cdot \varepsilon \varepsilon$
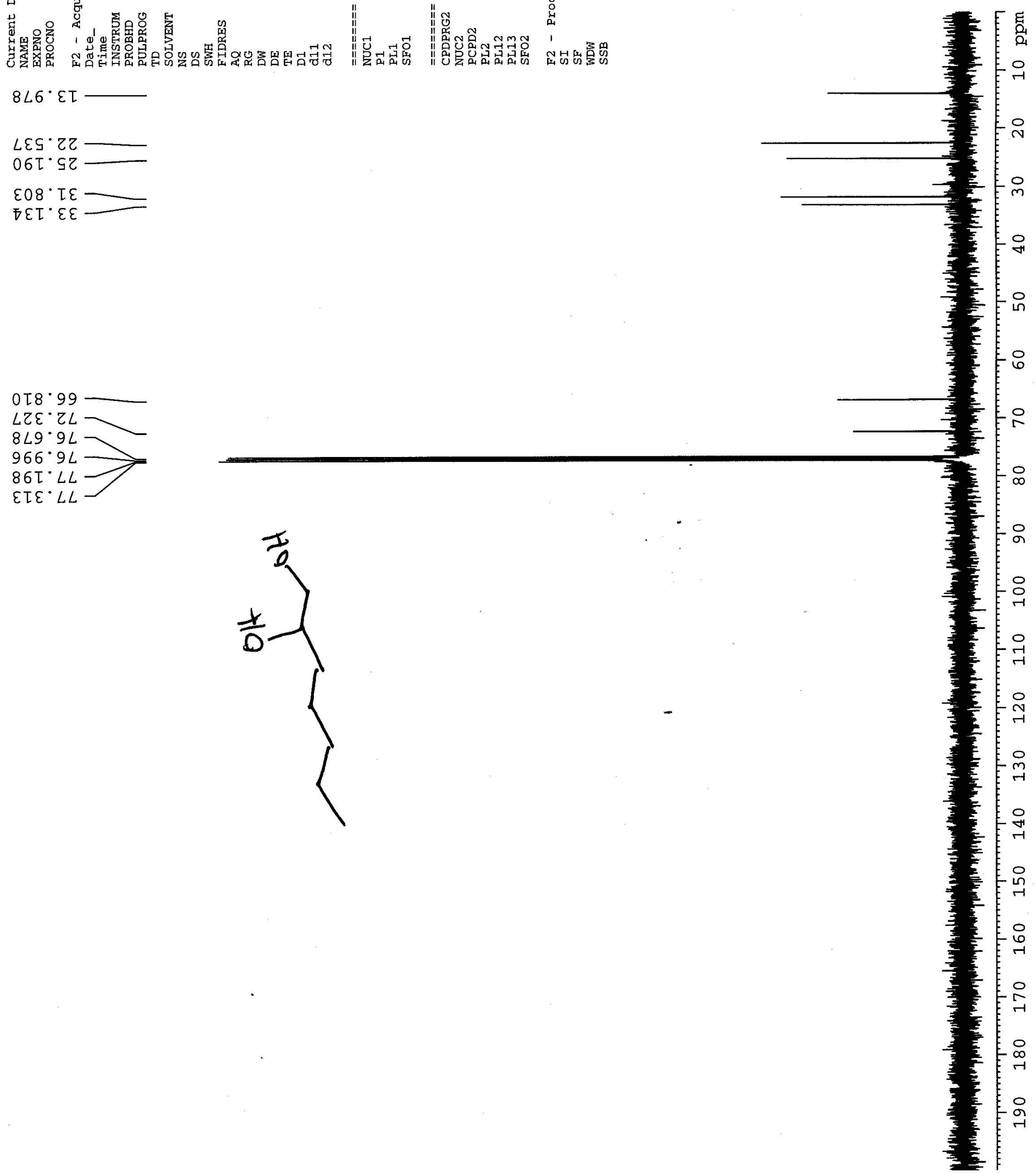

Page S - 16 

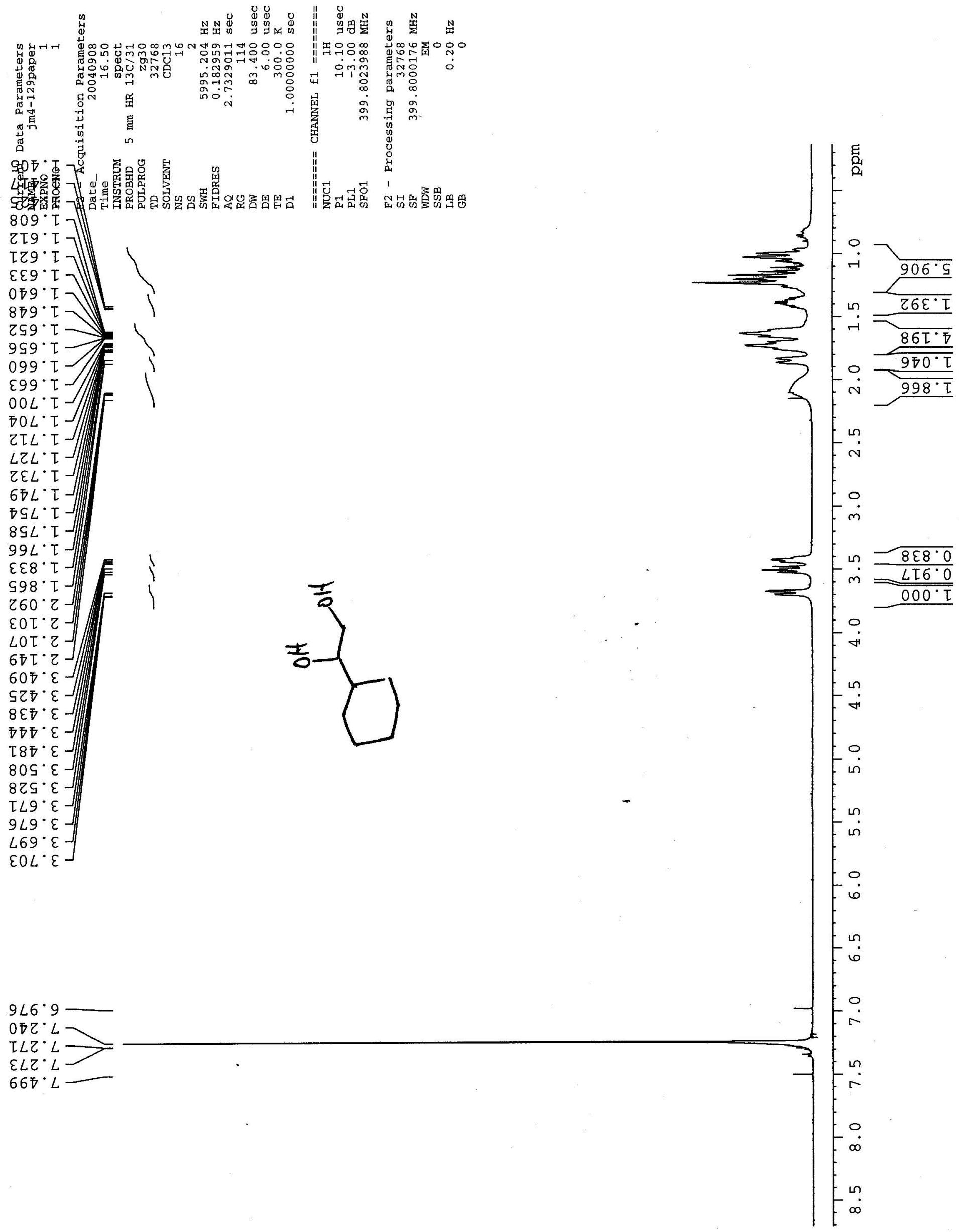

Page S - 17 

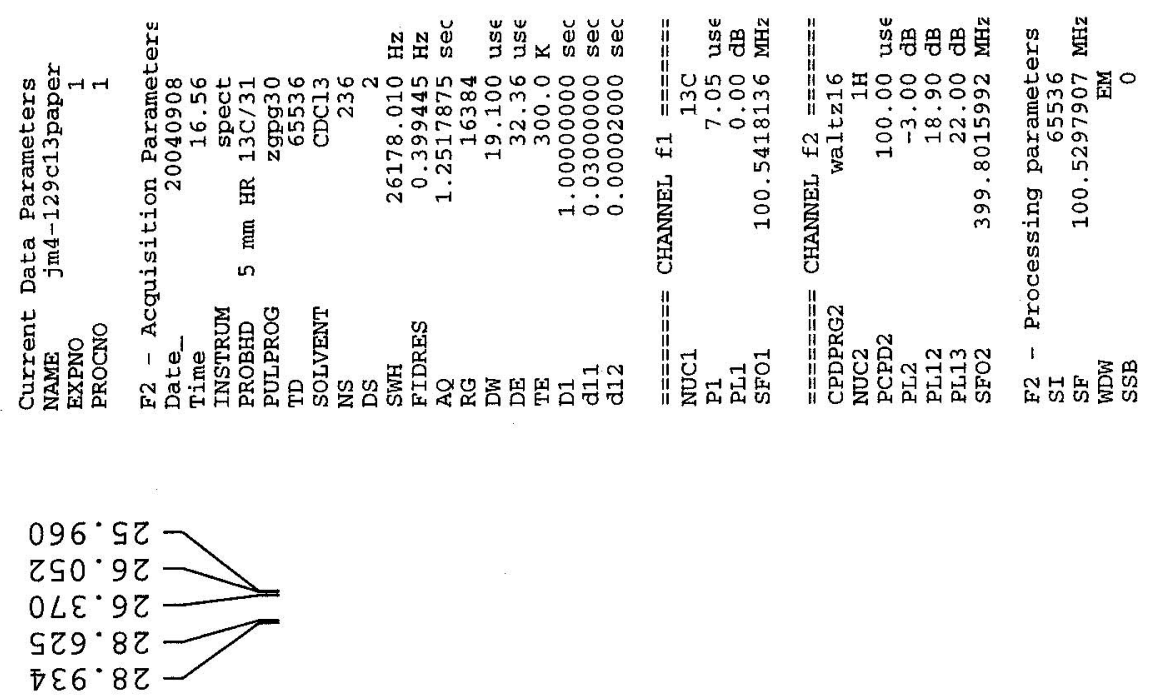

$97 L \cdot O \nabla$
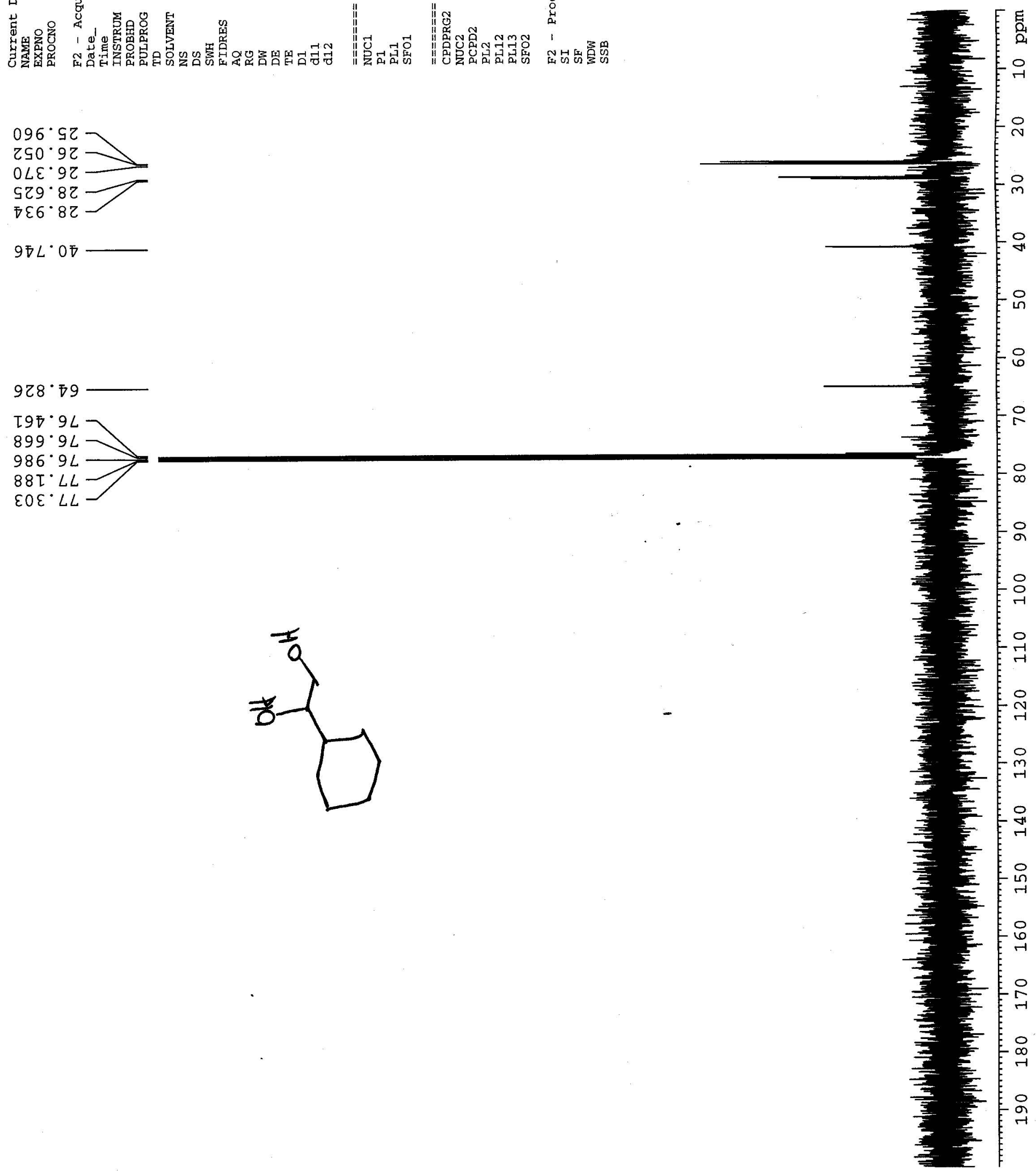

Page S - 18 


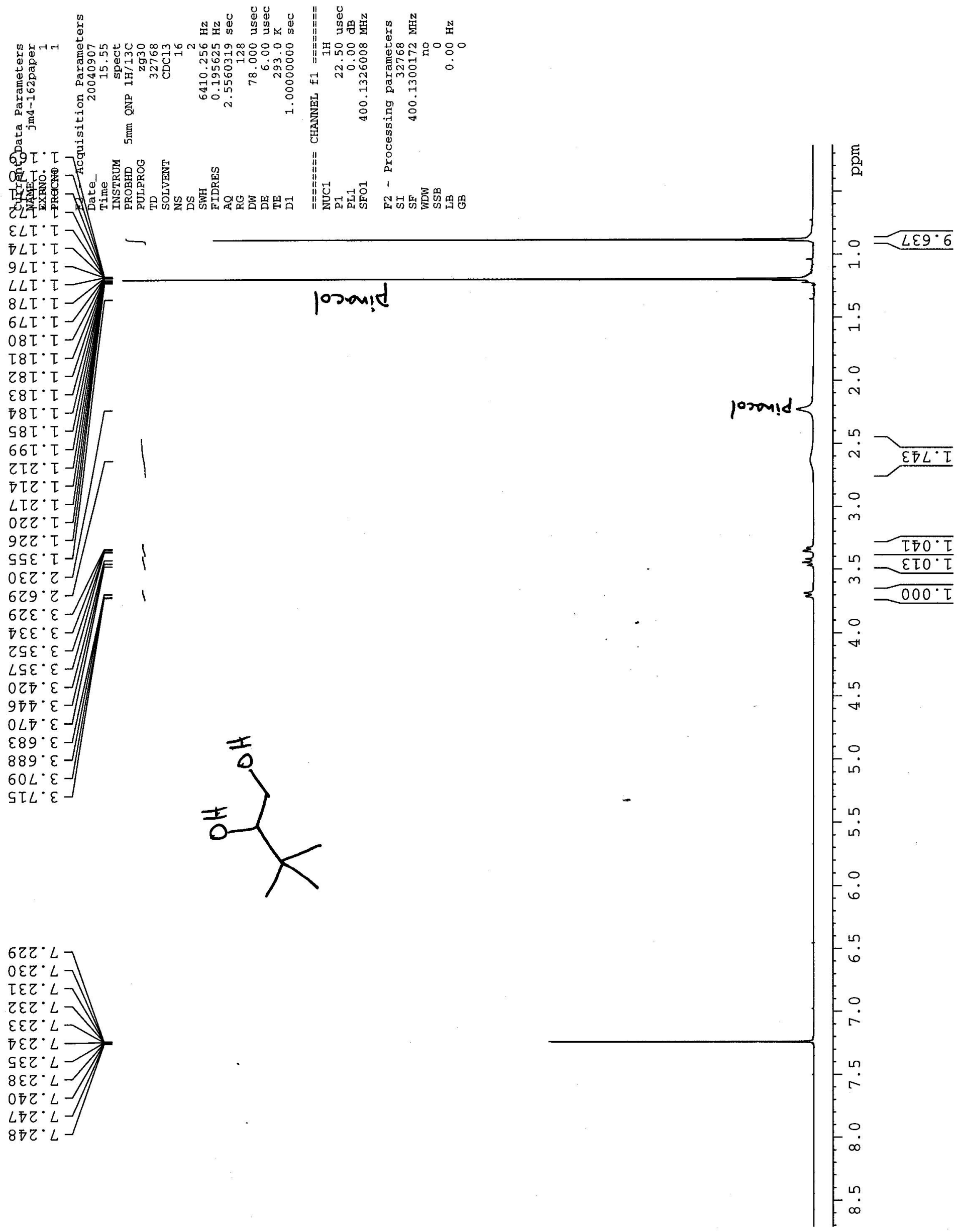

Page S - 19 


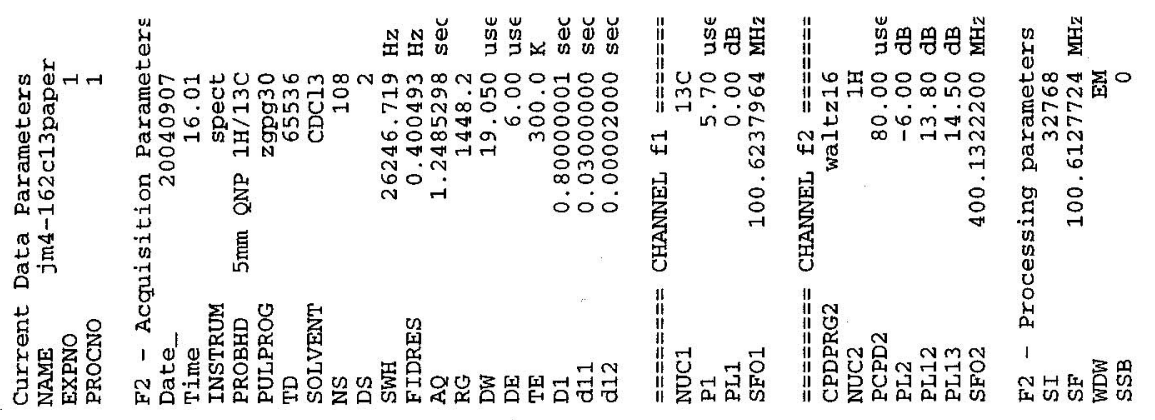

$86 L^{\circ} 2 \square$
$868^{\circ} \mathrm{9}$

$\tau\left\ulcorner\varsigma^{*} \varepsilon \varepsilon\right.$

$790^{\circ} \varepsilon 9$

$\overline{790^{\circ}} \mathrm{s} L$

$\varepsilon 89^{\circ} 9 L$

TOO $\angle L$

$\varepsilon 0 \zeta^{\circ} L L$

$899^{\circ} 6 L$
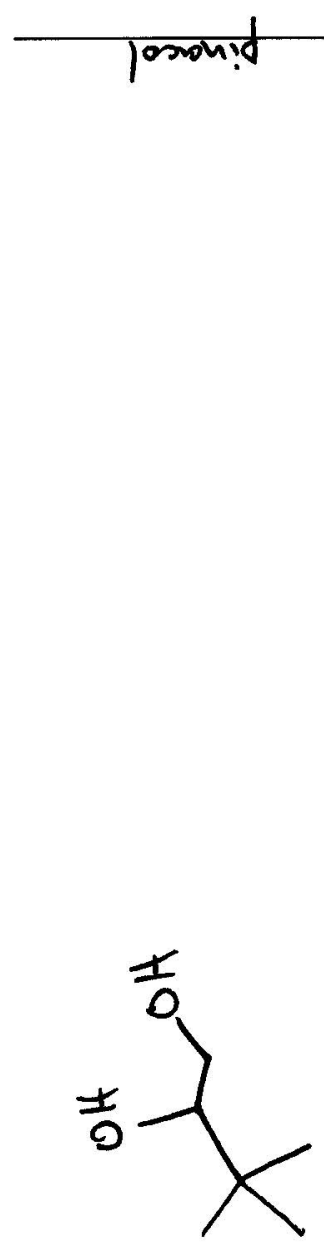

Page S - 20 

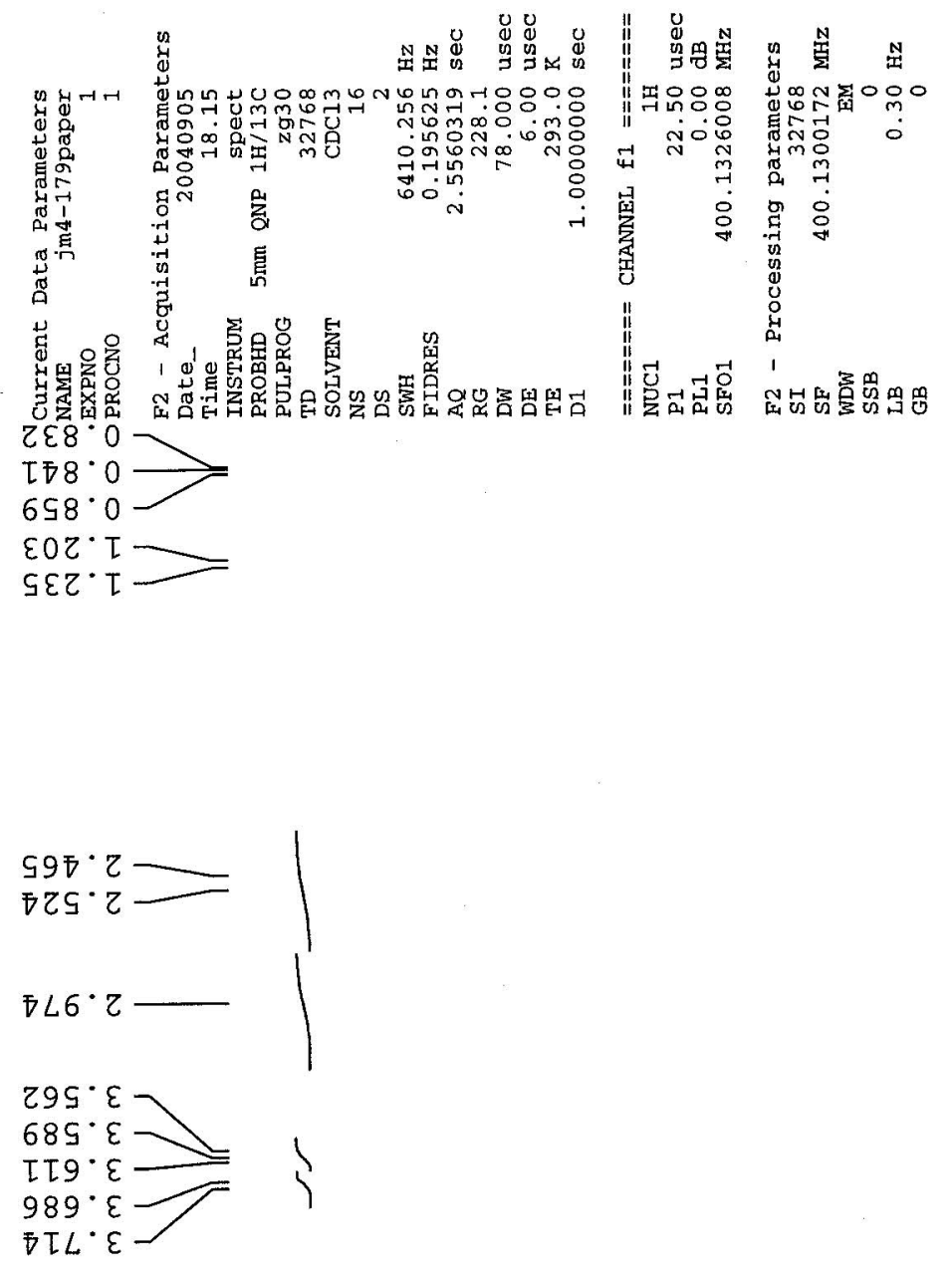

$\angle S L \cdot \nabla \longrightarrow$
$9 L L \cdot \nabla \longrightarrow$
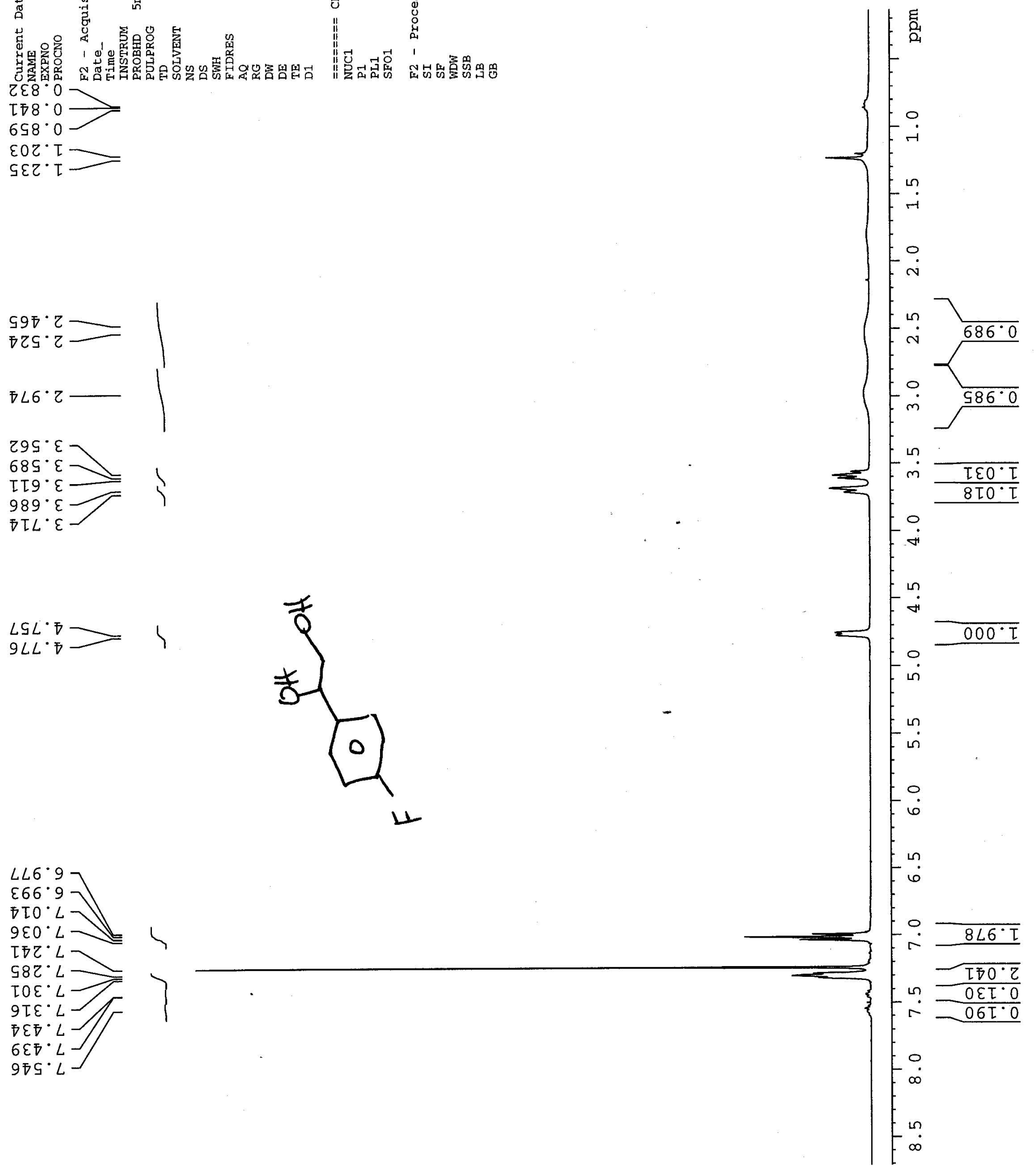

Page S - 21 

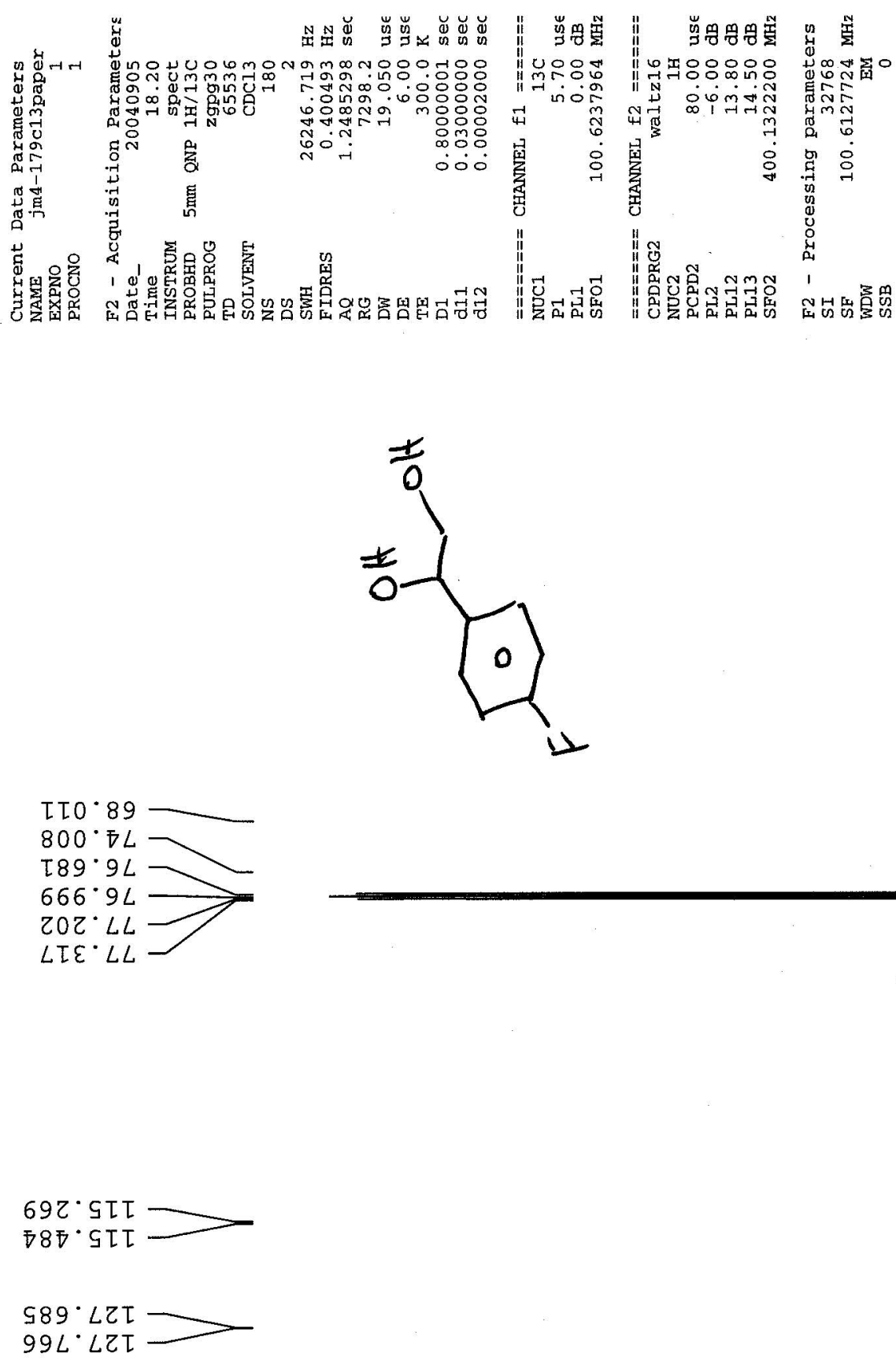

SLI.9EI $90 Z \cdot 9 \varepsilon \tau$

66 ' โ9 I

$9 ธ 9^{\circ} \varepsilon 9 \tau$

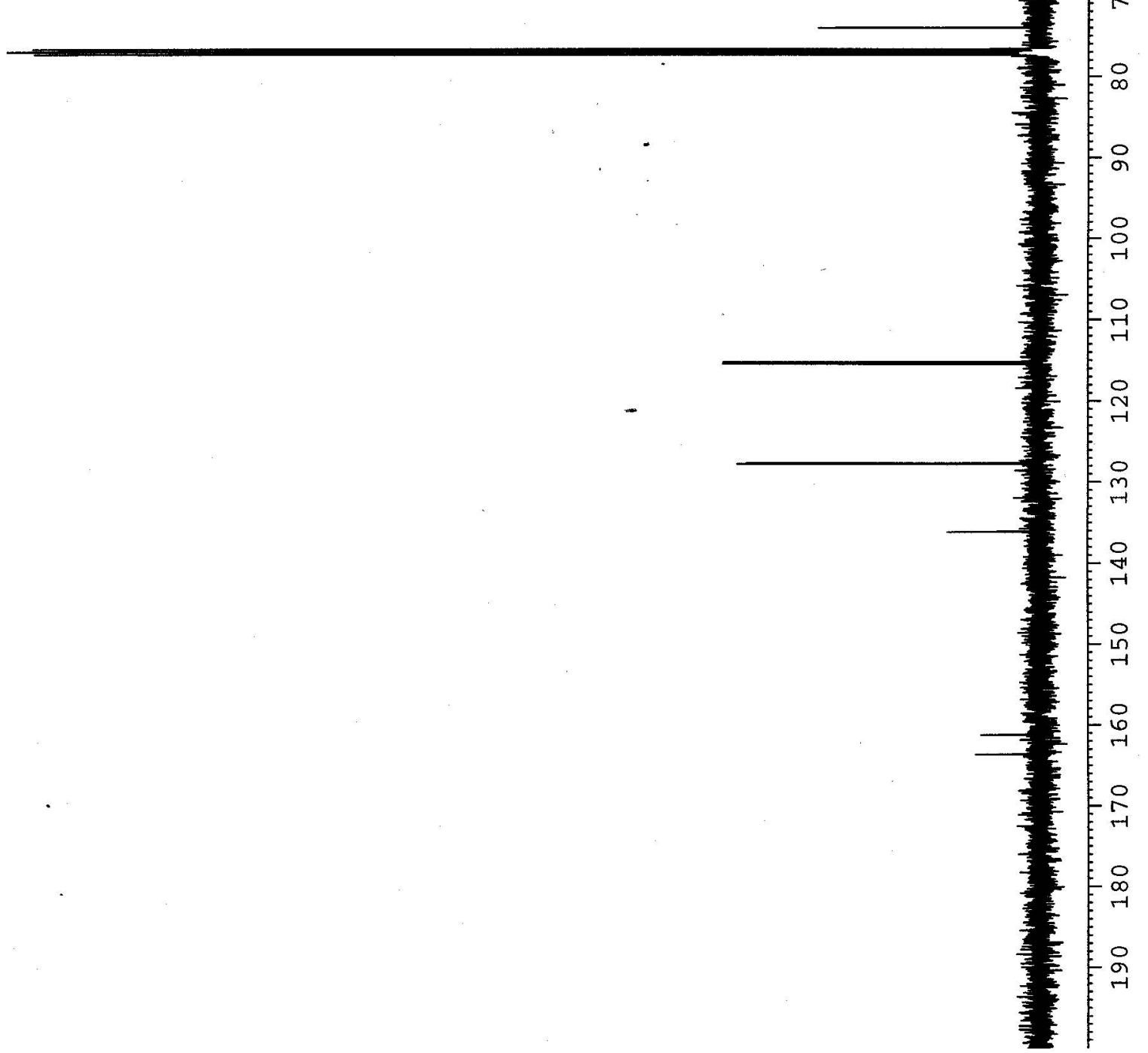

Page S - 22 